\title{
Model Selection and Hypothesis Testing for Large-Scale Network Models with Overlapping Groups
}

\author{
Tiago P. Peixoto* \\ Institut für Theoretische Physik, Universität Bremen, Hochschulring 18, D-28359 Bremen, Germany
}

(Received 21 October 2014; published 25 March 2015)

\begin{abstract}
The effort to understand network systems in increasing detail has resulted in a diversity of methods designed to extract their large-scale structure from data. Unfortunately, many of these methods yield diverging descriptions of the same network, making both the comparison and understanding of their results a difficult challenge. A possible solution to this outstanding issue is to shift the focus away from ad hoc methods and move towards more principled approaches based on statistical inference of generative models. As a result, we face instead the more well-defined task of selecting between competing generative processes, which can be done under a unified probabilistic framework. Here, we consider the comparison between a variety of generative models including features such as degree correction, where nodes with arbitrary degrees can belong to the same group, and community overlap, where nodes are allowed to belong to more than one group. Because such model variants possess an increasing number of parameters, they become prone to overfitting. In this work, we present a method of model selection based on the minimum description length criterion and posterior odds ratios that is capable of fully accounting for the increased degrees of freedom of the larger models and selects the best one according to the statistical evidence available in the data. In applying this method to many empirical unweighted networks from different fields, we observe that community overlap is very often not supported by statistical evidence and is selected as a better model only for a minority of them. On the other hand, we find that degree correction tends to be almost universally favored by the available data, implying that intrinsic node proprieties (as opposed to group properties) are often an essential ingredient of network formation.
\end{abstract}

DOI: 10.1103/PhysRevX.5.011033

\section{INTRODUCTION}

Many networks possess nontrivial large-scale structures such as communities [1,2], core peripheries [3,4], bipartitions [5], and hierarchies [6,7]. These structures presumedly reflect the organizational principles behind network formation. Furthermore, their detection can be used to predict missing links $[6,8]$ or detect spurious ones [8], as well as determine the robustness of the system to failure or intentional damage [9], the outcome of the spread of epidemics [10] and functional classification [11], among many other applications. The detail with which such modular features are both represented and detected reflects directly on the quality of these tasks. However, the methods of uncovering such structures in empirical data so far proposed are very different in their suitability to the aforementioned tasks. Many authors have constructed algorithms that attempt to divide the network into groups according to some metric devised

\footnotetext{
*tiago@itp.uni-bremen.de
}

Published by the American Physical Society under the terms of the Creative Commons Attribution 3.0 License. Further distribution of this work must maintain attribution to the author(s) and the published article's title, journal citation, and DOI.
Subject Areas: Complex Systems, Interdisciplinary Physics, Statistical Physics

specifically for this purpose. Examples of this include modularity [12], betweenness [13], link similarity [14], clique percolation [15], encoding of random walks [16], and many more [2]. Unfortunately, many of these methods will result in diverging descriptions for the same network. Furthermore, the information they obtain cannot be easily used to generalize the data and make predictions [6,8]. Alternatively, other authors have focused on constructing generative models that encode the large-scale structure as parameters, which can then be inferred from empirical data (see, e.g., Refs. [17-20]). These methods not only represent a more principled and rigorous stance, but they can also demonstrably overcome inherent limitations of more ad hoc methods [7]. Furthermore, they can be used to generalize the data and make predictions $[6,8]$. Both approaches, however, suffer from a common fundamental problem, namely, the difficulty in deciding which detection method or generative model provides a more appropriate description of a given network. This issue tends to escalate as more elaborate models and methods are developed, including features such as degree correction [19], community overlap [14,15,18,20], hierarchical structure [6,7,21], self-similarity [22,23], bipartiteness [5], edge and node correlates [24,25], social tiers [26], multilayer structure [27], and temporal information [28], to name only a 
few. Although such developments are essential, they should be made with care since increasing the complexity of the network description may lead to artificial results caused by overfitting. While this is a well-understood phenomenon when dealing with independent data or time series, open problems remain when the empirical data are a network, for which many common assumptions no longer hold and the usual methods perform very poorly [29]. This problem is significantly exacerbated when methods are used which make no attempt to assess the statistical significance of the results. Unfortunately, most methods that are not based on generative models fall into this class. Although for certain specially constructed examples some direct connections between statistical inference and ad hoc methods can be made [30,31], and in the case of some spectral methods a much deeper connection seems to exist [32,33], they still inherently lack the capacity to reliably distinguish signal from noise. Furthermore - what is perhaps even more importantthese different methods cannot easily be compared to each other. For example, suppose that for the same network a nonoverlapping partition is found by compressing random walks, another overlapping partition is obtained with clique percolation, and yet another with a local method based on link similarity (all of which are methods not based on generative models). Most of the time, these three partitions will be very different, and yet there is no obvious way to decide which one is a more faithful representation of the network. Although methods such as network benchmarks [34-36] and perturbation analysis [37] have been developed in order to alleviate this issue, they have only limited applicability to the larger problem. Namely, network benchmarks cannot be used when an appropriate representation of an empirical network is not known and if one wants to decide, for instance, if the network possesses overlapping groups or not. In a similar vein, perturbation analysis provides information about the significance of results originating from a single algorithm, which cannot be directly used to compare two very different ones.

On the other hand, the situation is different if one focuses on generative models alone. Since in this context the same problem is posed in a probabilistic framework, comparison between models is possible, even if the models are very different. And since models can be designed to accommodate arbitrary topological features, we lose no explanatory power when comparing to the ad hoc approaches. We show in this work that this central issue can be tackled in a consistent and principled manner by performing model selection based on statistical evidence. In particular, we employ the minimum description length principle (MDL) $[38,39]$, which seeks to minimize the total information necessary to describe the observed data as well as the model parameters. This can be equivalently formulated as the maximization of a Bayesian posterior likelihood which includes noninformative priors on the parameters, from which a posterior odds ratio between different hypotheses can be computed, yielding a degree of confidence for a model to be rejected in favor of another. We focus on the stochastic block model as the underlying generative model, as well as variants that include degree correction and mixed memberships. We show that with these models, MDL can be used to produce a very efficient algorithm that scales well for very large networks and with an arbitrarily large number of groups. Furthermore, we employ the method to a wide variety of empirical network data sets, and we show that community overlaps are seldom selected as the most appropriate model. This casts doubt on the claimed pervasiveness of group overlaps $[14,15]$, obtained predominantly with nonstatistical methods, which should perhaps be interpreted as an artifact of using methods with more degrees of freedom, instead of an underlying property of many systems - at least as long as there is a lack of corroborating evidence supporting the overlap (such as, potentially, edge weights [25,40] or multilayer structure [27], which we do not consider here). On the other hand, we find that degree correction tends to be selected for a significant majority of systems, implying that individual node "fitness" that is not uniformly inherited by group membership is a fundamental aspect of network formation.

This paper is divided as follows. In Sec. II, we present the generative models considered, and in Sec. III, we describe the model selection procedure based on MDL. In Sec. IV, we present the results for a variety of empirical networks. In Sec. V, we analyze the general identifiability limits of the overlapping models, and in Sec. VI, we describe in detail the inference algorithm used. In Sec. VII, we finalize our work with a discussion.

\section{GENERATIVE MODELS FOR NETWORK STRUCTURE}

A generative model is one which attributes to each possible graph $G$ a probability $P(G \mid\{\theta\})$ for it to be observed, conditioned on some set of parameters $\{\theta\}$. Here, we will be restricted to discrete uniform models, where specific choices of $\{\theta\}$ prohibit some graphs from occurring, but those which are allowed to occur have the same probability. For these models, we can write $P(G \mid\{\theta\})=1 / \Omega(\{\theta\})=e^{-\mathcal{S}(G \mid\{\theta\})}$, with $\Omega(\{\theta\})$ being the total number of possible graphs compatible with a given choice of parameters, and $\mathcal{S}(G \mid\{\theta\})=\ln \Omega(\{\theta\})$ is the entropy of this constrained ensemble [41,42]. In order to infer the parameters $\{\theta\}$ via maximum likelihood, we need to maximize $P(G \mid\{\theta\})$ or, equivalently, minimize $\mathcal{S}(G \mid\{\theta\})$. This approach, however, cannot be used if the order of the model is unknown, i.e., the number of degrees of freedom in the parameter set $\{\theta\}$, since choices with higher order will almost always increase $P(G \mid\{\theta\})$, resulting in overfitting. For the same reason, maximum likelihood cannot be used to distinguish between models belonging to different classes since models with larger degrees of freedom will inherently lead to larger 
likelihoods. In order to avoid overfitting, one needs to maximize instead the Bayesian posterior probability $P(\{\theta\} \mid G)=P(G \mid\{\theta\}) P(\{\theta\}) / P(G)$, with $P(G)$ being a normalizing constant. The prior probability $P(\{\theta\})$, which encodes our a priori knowledge of the parameters (if any), should inherently become smaller if the number of degrees of freedom increases. We will also be restricted to discrete parameters with uniform prior probabilities, so that $P(\{\theta\})=e^{-\mathcal{L}(\{\theta\})}$, with $\mathcal{L}(\{\theta\})$ being the entropy of the ensemble of possible parameter choices. We can thus write the total posterior likelihood as $P(\{\theta\} \mid G)=e^{-\Sigma} / P(G)$, with $\Sigma=\mathcal{L}(\{\theta\})+\mathcal{S}(G \mid\{\theta\})$. The value $\Sigma$ is the description length of the data [38,39], i.e., the total amount of information required to describe the observed data conditioned on a set of parameters as well as the parameter set itself [43]. Hence, if we maximize $P(\{\theta\} \mid G)$, we are automatically finding the parameter choice that compresses the data most since it will also minimize its description length $\Sigma$. Because of this, there is no difference between specifying probabilistic models for both $G$ and $\{\theta\}$ or encoding schemes that quantify the amount of information necessary to describe both. In the following, we will make use of both terminologies interchangeably, whenever most appropriate.

\section{A. Overlapping model without degree correction}

The main feature we want to consider in our generative model is the existence of well-defined groups of nodes, which are connected to other groups with arbitrary probabilities, such that nodes belonging to the same group play a similar role in the large-scale network structure. We also want to include the possibility of nodes belonging to more than one group and, in so doing, inherit the topological properties of all groups to which they belong. In order to implement this, we consider a simple variation of the stochastic block model $[17,44-46]$ with $N$ nodes and $E$ edges, where the nodes can belong to different groups. Hence, to each node $i$, we attribute a binary mixture vector $\vec{b}_{i}$ with $B$ entries, where a given entry $b_{i}^{r} \in\{0,1\}$ specifies whether or not the node belongs to block $r \in[1, B]$. In addition to this overlapping partition, we simply define the edge-count matrix $\left\{e_{r s}\right\}$, which specifies how many edges are placed between nodes belonging to blocks $r$ and $s$ (or twice that number for $r=s$, for convenience of notation), where we have $\sum_{r s} e_{r s}=2 E$. This simple definition allows one to generate a broad variety of overlapping patterns, which are not confined to purely assortative structures, and the nonoverlapping model can be recovered as a special case, simply by putting each node in a single group.

The likelihood of observing a given graph with the above constraints is simply $P\left(G \mid\left\{\vec{b}_{i}\right\},\left\{e_{r s}\right\}\right)=1 / \Omega\left(\left\{\vec{b}_{i}\right\}\right.$, $\left.\left\{e_{r s}\right\}\right)$, where $\Omega\left(\left\{\vec{b}_{i}\right\},\left\{e_{r s}\right\}\right)$ is the number of possible graphs, and $\mathcal{S}_{t}=\ln \Omega\left(\left\{\vec{b}_{i}\right\},\left\{e_{r s}\right\}\right)$ is the associated ensemble entropy. In this construction, the existence of multiple edges is allowed. However, the placement of multiple edges between nodes of blocks $r$ and $s$ should occur with a probability proportional to $O\left(e_{r s} / n_{r} n_{s}\right)$, where $n_{r}$ is the number of nodes which belong to block $r$, i.e., $n_{r}=\sum_{i} b_{i}^{r}$ (note that $\sum_{r} n_{r} \geq N$ ). Since here we are predominantly interested in the sparse situation where $e_{r s} \sim O\left(N / B^{2}\right)$ and $n_{r} \sim O(N / B)$, the probability of observing parallel edges will decay as $O(1 / N)$ and hence can be neglected in the large network limit. Making use of this simplification, we may approximately count all possible graphs generated by the parameters $\left\{\vec{b}_{i}\right\},\left\{e_{r s}\right\}$ as the number of graphs where each distinct membership of a single node is considered to be a different node with a single membership. This corresponds to an augmented graph generated via a nonoverlapping block model with $N^{\prime}=\sum_{r} n_{r}$ nodes, where $N^{\prime} \geq N$, but with the same matrix $\left\{e_{r s}\right\}$, for which the entropy is [42]

$$
\mathcal{S}_{t} \simeq E-\frac{1}{2} \sum_{r s} e_{r s} \ln \left(\frac{e_{r s}}{n_{r} n_{s}}\right),
$$

where $n_{r} n_{s} \gg e_{r s}$ was assumed. Under this formulation, we recover trivially the single-membership case simply by assigning each node to a single group since Eq. (1) remains the same in that special case. It is possible to remove the approximation that no parallel edges occur, by defining the model somewhat differently, as shown in Appendix B 1, in which case Eq. (1) holds exactly as long as no parallel edges are observed.

Like its nonoverlapping counterpart, the block model without degree correction assumes that nodes belonging to the same group will receive approximately the same number of edges of that type. Hence, when applied to empirical data, the modules discovered will also tend to have this property. This means that if the graph possesses large degree variability, the groups inferred will tend to correspond to different degree classes [19]. In a similar vein, if a node belongs to more than one group, it will also tend to have a total degree that is larger than nodes that belong to either group alone since it will receive edges of each type in an independent fashion. In other words, the group intersections are expected to be strictly denser than the nonoverlapping portions of each group. Note that, in this respect, this model differs from other popular ones, such as the mixed membership stochastic block model (MMSBM) [18], where the density at the intersections is the weighted average of the groups (see Appendix B 1).

\section{B. Overlapping model with degree correction}

In the preceding model, nodes that belong to the same group mixture receive, on average, the same number of connections. This means that the group membership is the only factor regulating the propensity of a given node to receive links. An alternative possibility, formulated by Karrer et al. [19], is to consider that the nodes have 
individual propensities to connect themselves, which are not necessarily correlated with their group memberships. Therefore, in this "degree-corrected" model, nodes of the same group are allowed to possess very different degrees. It has been demonstrated in Ref. [19] that this model yields more intuitive partitions for many empirical networks, suggesting that these intrinsic propensities may be a better model for these systems. In an analogous manner, a multiple membership version of the stochastic block model with degree correction can be defined. This definition can be achieved simply by specifying, in addition to the overlapping partition $\left\{\vec{b}_{i}\right\}$, the number of half-edges incident on a given node $i$ which belong to group $r$, i.e., $k_{i}^{r}$. The combined labeled degree of a node $i$ is denoted $\vec{k}_{i}=\left\{k_{i}^{r}\right\}$. Given this labeled degree sequence, one can simply use the same edge count matrix $\left\{e_{r s}\right\}$ as before to generate the graph. If we again make the assumption that the occurrence of parallel edges can be neglected, the total number of graphs fulfilling these constraints is approximately equal to the nonoverlapping ensemble where each set of half-edges incident on any given node $i$ that belongs to the same group $r$ is considered as an individual node with degree $k_{i}^{r}$, for which the ensemble entropy is [42]

$$
\mathcal{S}_{d} \simeq-E-\frac{1}{2} \sum_{r s} e_{r s} \ln \left(\frac{e_{r s}}{e_{r} e_{s}}\right)-\sum_{i r} \ln k_{i}^{r !}
$$

where $e_{r s}\left(\left\langle k^{2}\right\rangle_{r}-\langle k\rangle_{r}\right)\left(\left\langle k^{2}\right\rangle_{s}-\langle k\rangle_{s}\right) /\langle k\rangle_{r}^{2}\langle k\rangle_{s}^{2} \ll n_{r} n_{s}$ has been assumed. Similarly to the non-degree-corrected case, it is possible to remove the approximation that no parallel edges occur by using a "Poisson" version of the model, as is shown in Appendix B 2. Under this formulation, it can be shown that this model is equivalent to the one proposed by Ball et al. [20], although here we keep track of the individual labels on the half-edges as latent variables, instead of their probabilities.

Since we incorporate the labeled degree sequence as model parameters, nodes that belong to the same group can have arbitrary degrees. Furthermore, since the same applies to nodes that belong simultaneously to more than one group, the overlaps between groups are neither preferably dense nor sparse; it all depends on the parameters $\left\{\vec{k}_{i}\right\}$.

\section{MODEL SELECTION}

As discussed previously, in order to perform model selection, it is necessary to include the information needed to describe the model parameters, in addition to the data. The parameters that need to be described are the overlapping partition $\left\{\vec{b}_{i}\right\}$ and the edge counts $\left\{e_{r s}\right\}$, and in the case of the degree-corrected model, we also need to the describe the labeled degree sequence $\left\{\vec{k}_{i}\right\}$.

When choosing an encoding for the parameters (via a particular generative process), we need to avoid redundancy and describe them as parsimoniously as possible while at the same time averting biases by being noninformative. In the following, we systematically employ twolevel Bayesian hierarchies, where discrete prior distributions are parametrized via generic counts, which are themselves sampled from uniform nonparametric hyperpriors.

\section{A. Overlapping partition, $\left\{\overrightarrow{\boldsymbol{b}}_{\boldsymbol{i}}\right\}$}

In order to specify the partition $\left\{\vec{b}_{i}\right\}$, we assume that all different $2^{B}-1$ mixtures are not necessarily equally likely, and furthermore, the sizes $d_{i}=\sum_{r} b_{i}^{r}$ of the mixtures are also not a priori assumed to follow any specific distribution. More specifically, we consider the mixtures to be the outcome of a generative process with two steps. We first generate the local mixture sizes $\left\{d_{i}\right\}$ from a nonparametric distribution. Then, given the mixture sizes, we generate the actual mixtures $\left\{\vec{b}_{i}\right\}$ for each corresponding subset of the nodes, again using a nonparametric distribution, conditioned on the mixture size.

The mixture sizes $\left\{d_{i}\right\}$ are sampled uniformly from the distribution with fixed counts $\left\{n_{d}\right\}$, where $n_{d}$ is the number of nodes with a mixture of size $d_{i}=d$, with a likelihood

$$
P\left(\left\{d_{i}\right\} \mid\left\{n_{d}\right\}\right)=\frac{\prod_{d} n_{d} !}{N !} .
$$

For the counts $\left\{n_{d}\right\}$, we assume a flat prior $P\left(\left\{n_{d}\right\}\right)=$ $1 /\left(\left(\begin{array}{l}D \\ N\end{array}\right)\right)$, where $D$ is the maximum value of $d$, and the denominator is the total number of different choices of $\left\{n_{d}\right\}$, with $\left(\left(\begin{array}{c}n \\ m\end{array}\right)\right)=\left(\begin{array}{c}n+m-1 \\ m\end{array}\right)$ being the total number of $m$ combinations with repetitions from a set of size $n$.

Then, for all $n_{d}$ nodes with the same value of $d_{i}=d$, we sample a sequence of $\left\{\vec{b}_{i}\right\}_{d}$ from a distribution with support $\left|\vec{b}_{i}\right|_{1} \equiv \sum_{r} b_{i}^{r}=d$ and with fixed counts $\left\{n_{\vec{b}}\right\}_{d}$, where $n_{\vec{b}}$ is the number of nodes belonging to a specific mixture $\vec{b}_{i}=\vec{b}$ of size $d$,

$$
P\left(\left\{\vec{b}_{i}\right\}_{d} \mid\left\{n_{\vec{b}}\right\}_{d}\right)=\frac{\prod_{|\vec{b}|_{1}=d} n_{\vec{b}} !}{n_{d} !} .
$$

For the counts themselves, we again assume a flat prior

$$
P\left(\left\{n_{\vec{b}}\right\}_{d} \mid n_{d}\right)=1 /\left(\left(\begin{array}{c}
B \\
d \\
n_{d}
\end{array}\right)\right),
$$

where the denominator enumerates the total number of $\left\{n_{\vec{b}}\right\}$ counts with $|\vec{b}|_{1}=d$.

The full posterior for the overlapping partition then becomes

$$
\begin{aligned}
P\left(\left\{\vec{b}_{i}\right\}\right)= & {\left[\prod_{d} P\left(\left\{\vec{b}_{i}\right\}_{d} \mid\left\{n_{\vec{b}}\right\}_{d}\right) P\left(\left\{n_{\vec{b}}\right\}_{d} \mid n_{d}\right)\right] } \\
& \times P\left(\left\{d_{i}\right\} \mid\left\{n_{d}\right\}\right) P\left(\left\{n_{d}\right\}\right),
\end{aligned}
$$


which corresponds to a description length $\mathcal{L}_{p}=$ $-\ln P\left(\left\{\vec{b}_{i}\right\}\right)$,

$$
\begin{aligned}
\mathcal{L}_{p}= & \ln \left(\left(\begin{array}{c}
D \\
N
\end{array}\right)\right)+\sum_{d} \ln \left(\left(\begin{array}{l}
B \\
d \\
n_{d}
\end{array}\right)\right) \\
& +\ln N !-\sum_{\vec{b}} \ln n_{\vec{b}} ! .
\end{aligned}
$$

Although it is possible to encode the partition in different ways (e.g., by sampling the membership to each group independently [47]), this choice makes no assumptions regarding the types of overlaps that are more likely to occur, either according to the number of groups to which a node may belong or the actual combination of groups-it is all left to be learned from data. In particular, it is not a priori assumed that if many nodes belong to two specific groups, then the overlap between these same groups will also contain many nodes. As desired, if the observed partition deviates from this pattern, this will be used to compress it further. Only if the observed partition falls squarely into this pattern will further compression be impossible, and we would have an overhead describing it using Eq. (6), when compared to an encoding that expects it a priori. However, one can also see that in the limit $n_{\vec{b}} \gg 1$, as the first two terms in Eq. (6) grow asymptotically only with $\ln N$ and $\ln n_{d}$, respectively, the whole description length becomes $\mathcal{L}_{p} \simeq N H\left(\left\{n_{\vec{b}} / N\right\}\right)$, where $H\left(\left\{p_{x}\right\}\right)$ is the entropy of the distribution $\left\{p_{x}\right\}$, which is the optimal limit. Hence, if we have a prior that better matches the observed overlap, the difference in description length compared to Eq. (6) will disappear asymptotically for large systems. Another advantage of this encoding is that it incurs no overhead when there are no overlaps at all (i.e., $D=1$ ), and in this case, the description length is identical to the nonoverlapping case,

$$
\mathcal{L}_{p}(D=1)=\ln \left(\left(\begin{array}{c}
B \\
N
\end{array}\right)\right)+\ln N !-\sum_{r} \ln n_{r} !
$$

as defined in Ref. [7].

\section{B. Labeled degree sequence, $\left\{\overrightarrow{\boldsymbol{k}}_{\boldsymbol{i}}\right\}$}

For the degree-corrected model, we need to describe the labeled degree sequence $\left\{\vec{k}_{i}\right\}$. We need to do so in a way that is compatible with the partition $\left\{\vec{b}_{i}\right\}$ described so far, and with the edge counts $\left\{e_{r s}\right\}$, which will restrict the average degrees of each type.

In order to fully utilize the partition $\left\{\vec{b}_{i}\right\}$, we describe for each distinct value of $\vec{b}_{i}=\vec{b}$, its individual degree sequence $\left\{\vec{k}_{i}\right\}_{\vec{b}}=\left\{\vec{k}_{i} \mid \vec{b}_{i}=\vec{b}\right\}$, via the counts $n_{\vec{k}}$, i.e., the number of nodes with mixture $\vec{b}_{i}=\vec{b}$ which possess labeled degree $\vec{k}_{i}=\vec{k}$. We do so in order to preserve the lack of preference for patterns involving the degrees in the overlaps between groups. Since the model itself is agnostic with respect to the density of the overlaps, not only does this choice remain consistent with this indifference, but also any existing pattern in the degree sequence in the overlaps will be used to construct a shorter description.

In addition, we must also consider the total number of half-edges of a given type $r$ incident on a partition $\vec{b}$, $e_{\vec{b}}^{r}=\sum_{\vec{k}} k_{r} n_{\vec{k}}^{\vec{b}}$, where $k_{r}$ is the element of $\vec{k}$ corresponding to group $r$, which must be compatible with the edge counts $\left\{e_{r s}\right\}$ via $e_{r}=\sum_{s} e_{r s}=\sum_{\vec{b}} e_{\vec{b}}^{r}$.

An overview of the generative process is as follows: We first consider the $e_{r}$ half-edges of each type $r$ and the nonempty $\left(n_{\vec{b}}>0\right)$ mixtures $\vec{b}$ that contain the same group $r$. We then distribute the labeled half-edges among these mixtures, obtaining the total number of labeled edges incident on each mixture, $\left\{e_{\vec{b}}^{r}\right\}$. This placement constrains the average degree of each type inside each mixture. Finally, given $\left\{e_{\vec{b}}^{r}\right\}$, we sample the actual labeled degree sequence on the nodes of each mixture.

We begin by first distributing all $e_{r}$ half-edges of type $r$ among all $m_{r}$ bins corresponding to each nonempty mixture $\vec{b}$ that contains the label $r$, i.e., $m_{r}=$ $\sum_{\vec{b}} b_{r}\left[n_{\vec{b}}>0\right]$. The total number of such partitions is simply $\left(\left(\begin{array}{c}m_{r} \\ e_{r}\end{array}\right)\right.$, and hence the likelihood for $\left\{e_{\vec{b}}^{r}\right\}$ becomes

$$
P\left(\left\{e_{\vec{b}}^{r}\right\} \mid\left\{e_{r s}\right\},\left\{\vec{b}_{i}\right\}\right)=\left(\left(\begin{array}{c}
m_{r} \\
e_{r}
\end{array}\right)\right)^{-1} .
$$

Given $\left\{e_{\vec{b}}^{r}\right\}$, we need to distribute the labeled half-edges inside each partition to obtain each degree sequence. If we sample uniformly from all possible degree sequences fulfilling all necessary constraints, we have a likelihood for the degree sequence inside a mixture $\vec{b}$ given by

$$
P_{\vec{b}}^{(1)}\left(\left\{\vec{k}_{i}\right\}_{\vec{b}} \mid\left\{e_{\vec{b}}^{r}\right\},\left\{\vec{b}_{i}\right\}\right)=\prod_{r}\left(\left(\begin{array}{c}
n_{\vec{b}} \\
e_{\vec{b}}^{r}
\end{array}\right)\right)^{-1},
$$

where $\left(\left(\begin{array}{l}n \\ e\end{array}\right)\right)$ is the total number of (unlabeled) degree sequences with a total of $e$ half-edges incident on $n$ nodes. The corresponding description length would then be

$$
\mathcal{L}_{\vec{b}}^{(1)}=\sum_{r} \ln \left(\left(\begin{array}{c}
n_{\vec{b}} \\
e_{\vec{b}}^{r}
\end{array}\right)\right) .
$$

However, most degree sequences sampled this way will result in nodes with very similar degrees. Since we want to profit from degree variability, it is better to condition the description on the degree counts $\left\{n_{\vec{k}}^{\vec{b}}\right\}$, i.e., how many nodes with mixture $\vec{b}_{i}=\vec{b}$ possess labeled degree $\vec{k}_{i}=\vec{k}$. This alternative distribution is given by 


$$
P_{\vec{b}}^{(2)}\left(\left\{\vec{k}_{i}\right\}_{\vec{b}} \mid\left\{n_{\vec{k}}^{\vec{b}}\right\},\left\{\vec{b}_{i}\right\}\right)=\frac{\prod_{\vec{k}} n_{\vec{k}} !}{n_{\vec{b}} !}
$$

For the degree counts themselves, we choose a uniform prior $P\left(\left\{n_{\vec{k}}^{\vec{b}}\right\} \mid\left\{e_{\vec{b}}^{r}\right\},\left\{\vec{b}_{i}\right\}\right)=1 / \Xi_{\vec{b}}$, where $\Xi_{\vec{b}}$ is the enumeration of all possible $\left\{n_{\vec{k}}^{\vec{b}}\right\}$ counts that fulfill the constraints $\sum_{\vec{k}} n_{\vec{k}}^{\vec{b}}=n_{\vec{b}}$ and $\sum_{\vec{k}} k_{r} n_{\vec{k}}^{\vec{b}}=e_{\vec{b}}^{r}$. Unfortunately, this enumeration cannot be done easily in closed form. However, the maximum entropy ensemble where these constraints are enforced on average is analytically tractable, and as we show in Appendix $\mathrm{C}$, it can be well approximated by $\Xi_{\vec{b}}=\prod_{r \in \vec{b}} \Xi_{\vec{b}}^{r}$, where

$$
\ln \Xi_{\vec{b}}^{r} \simeq 2 \sqrt{\zeta(2) e_{\vec{b}}^{r}}
$$

and $\zeta(x)$ is the Riemann zeta function. The alternative description length therefore becomes

$$
\mathcal{L}_{\vec{b}}^{(2)}=\sum_{r \in \vec{b}} \ln \Xi_{\vec{b}}^{r}+\ln n_{\vec{b}} !-\sum_{\vec{k}} \ln n_{\vec{k}}^{\vec{b}} !
$$

This approximation with "soft" constraints should become asymptotically exact as the number of nodes becomes large, but otherwise it will deviate from the actual entropy. On the other hand, if the number of nodes is very small, describing the degree sequence via Eq. (13) may not provide a shorter description, even if computed exactly. In this situation, Eq. (10) may actually provide a shorter description of the degree sequence. We therefore compute both Eqs. (10) and (13) and choose whichever is shorter. Putting it all together, the complete posterior for the whole labeled degree sequence is

$$
\begin{aligned}
P\left(\left\{\vec{k}_{i}\right\} \mid\left\{e_{r s}\right\},\left\{\vec{b}_{i}\right\}\right)= & {\left[\prod_{\vec{b}} P_{\vec{b}}\left(\left\{\vec{k}_{i}\right\}_{\vec{b}} \mid\left\{e_{\vec{b}}^{r}\right\},\left\{\vec{b}_{i}\right\}\right)\right] } \\
& \times P\left(\left\{e_{\vec{b}}^{r}\right\} \mid\left\{e_{r s}\right\},\left\{\vec{b}_{i}\right\}\right),
\end{aligned}
$$

with $P_{\vec{b}}\left(\left\{\vec{k}_{i}\right\}_{\vec{b}} \mid\left\{e_{\vec{b}}^{r}\right\},\left\{\vec{b}_{i}\right\}\right)$ being the largest choice between $P_{\vec{b}}^{(1)}\left(\left\{\vec{k}_{i}\right\}_{\vec{b}} \mid\left\{e_{\vec{b}}^{r}\right\},\left\{\vec{b}_{i}\right\}\right)$ and $P_{\vec{b}}^{(2)}\left(\left\{\vec{k}_{i}\right\}_{\vec{b}} \mid\left\{n_{\vec{k}}^{\vec{b}}\right\}\right)$ $P\left(\left\{n_{\vec{k}}^{\vec{b}}\right\} \mid\left\{e_{\vec{b}}^{r}\right\},\left\{b_{i}\right\}\right)$. Therefore, the description length for the labeled degree sequence $\mathcal{L}_{\kappa}=-\ln P\left(\left\{\vec{k}_{i}\right\} \mid\left\{e_{r s}\right\}\right.$, $\left.\left\{b_{i}\right\}\right)$ becomes

$$
\mathcal{L}_{\kappa}=\sum_{r} \ln \left(\left(\begin{array}{c}
m_{r} \\
e_{r}
\end{array}\right)\right)+\sum_{\vec{b}} \min \left(\mathcal{L}_{\vec{b}}^{(1)}, \mathcal{L}_{\vec{b}}^{(2)}\right) .
$$

In the limit $n_{\vec{k}}^{\vec{b}} \gg 1$, we have that $\mathcal{L}_{\kappa} \simeq \sum_{\vec{b}} n_{\vec{b}} H\left(\left\{n_{\vec{k}}^{\vec{b}} / n_{\vec{b}}\right\}\right)$, and hence the degree sequences in each partition are described close to the optimum limit.

For the nonoverlapping case with $D=1$, the description length simplifies to

$$
\mathcal{L}_{\kappa}=\sum_{r} \min \left(\mathcal{L}_{r}^{(1)}, \mathcal{L}_{r}^{(2)}\right),
$$

with

$$
\begin{gathered}
\mathcal{L}_{r}^{(1)}=\ln \left(\left(\begin{array}{c}
n_{r} \\
e_{r}
\end{array}\right)\right), \\
\mathcal{L}_{r}^{(2)}=\ln \Xi_{r}+\ln n_{r} !-\sum_{k} \ln n_{k}^{r} !
\end{gathered}
$$

and $\ln \Xi_{r} \simeq 2 \sqrt{\zeta(2) e_{r}}$. For $n_{r} \gg 1$, we obtain $\mathcal{L}_{\kappa} \simeq$ $\sum_{r} n_{r} H\left(\left\{n_{k}^{r} / n_{r}\right\}\right)$. This approximation was used a priori in Ref. [7], but Eq. (16) is a more complete description length of the nonoverlapping degree sequence, and its use should be preferred. Hence, like the description length of the overlapping partition, the encoding above offers no overhead when the partition is nonoverlapping.

\section{Edge counts, $\left\{\boldsymbol{e}_{r s}\right\}$}

The final piece that needs to be described is the matrix of edge counts $\left\{e_{r s}\right\}$. We may view this set as an adjacency matrix of a multigraph with $B$ nodes and $E=\sum_{r s} e_{r s} / 2$ edges. The total number of such matrices is

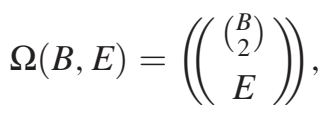

and if we assume that they are all equally likely, we have $P\left(\left\{e_{r s}\right\}\right)=1 / \Omega(B, E)$, and $\ln \Omega(B, E)$ can be used as the description length [48]. There are, however, two problems with this approach. First, this uniform distribution is unlikely to be valid since most observed networks still possess structure at the block level. Second, this assumption leads to a limit in the detection of small groups, with a maximum detectable number of groups scaling as $B_{\max } \sim \sqrt{N}$ [48]. Similarly to what we did for the node partition and the degree sequence, this can be solved by considering a generative model for the edge counts themselves, with its own set of hyperparameters. Since they correspond to a multigraph, a natural choice is the stochastic block model itself, which has its own set of edge counts, that can themselves be modeled by another stochastic block model with fewer nodes, and so on, recursively, until one has a model only one node and one group at the top. This nested stochastic block model was proposed in Ref. [7], where it was shown to reduce the resolution limit to $B_{\max } \sim N / \log N$, making it often significantly less relevant in practice. Furthermore, since the 
number of levels and the topology at each level is obtained by minimizing the overall description length, it corresponds to a fully nonparametric way of inferring the multilevel structure of networks. As shown in Ref. [7], if we denote the observed network to be at the level $l=0$ of the hierarchy, then the total description length is

$$
\Sigma=\mathcal{S}_{t / c}+\sum_{l=1}^{L} S_{m}\left(\left\{e_{r s}^{l}\right\},\left\{n_{r}^{l}\right\}\right)+\mathcal{L}_{t}^{l-1},
$$

with $\left\{e_{r s}^{l}\right\},\left\{n_{r}^{l}\right\}$ describing the block model at level $l$, where

$$
\mathcal{S}_{m}=\sum_{r>s} \ln \left(\left(\begin{array}{c}
n_{r} n_{s} \\
e_{r s}
\end{array}\right)\right)+\sum_{r} \ln \left(\left(\begin{array}{c}
\left.\left(\begin{array}{c}
n_{r} \\
2
\end{array}\right)\right) \\
e_{r r} / 2
\end{array}\right)\right)
$$

is the entropy of the corresponding multigraph ensemble and

$$
\mathcal{L}_{t}^{l}=\ln \left(\left(\begin{array}{c}
B_{l} \\
B_{l-1}
\end{array}\right)\right)+\ln B_{l-1} !-\sum_{r} \ln n_{r}^{l} !
$$

is the description length of the node partition at level $l>0$. For the level $l=0$, we have $\mathcal{L}_{t}^{0}=\mathcal{L}_{p}$, given by Eq. (6), or $\mathcal{L}_{t}^{0}=\mathcal{L}_{p}+\mathcal{L}_{\kappa}$ for the degree-corrected model.

Note that here we use the single-membership nondegree-corrected model in the upper layers. This method could be modified to include arbitrary mixtures of degree correction and multiple membership, but we stick with this formulation for simplicity.

\section{Significance levels}

By minimizing the description length $\Sigma$, we select the model that is most favored given the evidence in the data. But in some situations, one is not merely interested in a binary answer regarding which of two model choices is best, but instead, one would like to be able to rule out alternative models with some degree of confidence. In this case, a level of significance can be obtained by performing a Bayesian hypothesis test based on the ratio of posterior likelihoods. In this context, there are different hypotheses that can be tested. For instance, one could ask whether the entire class of non-degree-corrected overlapping models (NDCO) is favored in comparison to the class of nonoverlapping degree-corrected models (DC). This can be done by computing the posterior distribution for each model class $\mathcal{H} \in\{\mathrm{NDCO}, \mathrm{DC}\}$,

$$
P(\mathcal{H} \mid G)=\frac{\sum_{\theta} P(G \mid \theta, \mathcal{H}) P(\theta) P(\mathcal{H})}{P(G)}
$$

where $\theta$ is shorthand for the entire set of model parameters [i.e., $\theta=\left(\left\{\vec{b}_{i}\right\},\left\{e_{r s}\right\}\right)$ for $\mathcal{H}=\mathrm{NDCO}$, and $\theta=\left(\left\{b_{i}\right\}\right.$, $\left.\left\{e_{r s}\right\},\left\{k_{i}\right\}\right)$ for $\left.\mathcal{H}=\mathrm{DC}\right]$, with $P(\mathcal{H})$ being the prior belief we have that supports a given hypothesis, and $P(G)$ is a normalizing constant. The standard way in Bayesian statistics to evaluate the relative evidence supporting (or rejecting) hypothesis $\mathcal{H}_{1}$ over $\mathcal{H}_{2}$ is via the posterior odds ratio [49]

$$
\Lambda=\frac{P\left(\mathcal{H}_{1} \mid G\right)}{P\left(\mathcal{H}_{2} \mid G\right)}=\frac{\sum_{\theta} P\left(G \mid \theta, \mathcal{H}_{1}\right) P(\theta)}{\sum_{\theta} P\left(G \mid \theta, \mathcal{H}_{2}\right) P(\theta)} \frac{P\left(\mathcal{H}_{1}\right)}{P\left(\mathcal{H}_{2}\right)} .
$$

However, there are two issues with this approach. First, computing the sum over all parameter choices is intractable in this context since it involves summing over all possible overlapping or nonoverlapping partitions. Second, and more importantly, this might not be the answer that is more relevant. If one obtains two model parametrizations by minimizing the description length as described in the previous section, with the two results belonging to different model classes, one would be more interested in selecting or rejecting between these two particular choices, not necessarily the overall class to which they belong. Although the description length itself already provides a means to select the best alternative, one would be interested in obtaining a confidence level for this particular decision. This is a different sort of hypothesis test than the one above, but it can be performed analogously. Since the result of the minimization of the description length is the (possibly overlapping) partition of the network, our hypothesis is a combination of the model class that we were using and the particular partition that was found. The posterior probability attributed to this hypothesis is therefore

$$
P\left(\left\{\vec{b}_{i}\right\}, \mathcal{H} \mid G\right)=\frac{P\left(G \mid\left\{\vec{b}_{i}\right\}, \mathcal{H}\right) P\left(\left\{\vec{b}_{i}\right\} \mid \mathcal{H}\right) P(\mathcal{H})}{P(G)},
$$

where, again, $P(G)$ is a normalization constant. The marginal likelihood $P\left(G \mid\left\{\vec{b}_{i}\right\}, \mathcal{H}\right)$ is obtained by summing over the remaining model parameters. In the case of the overlapping degree-corrected model $(\mathcal{H}=\mathrm{DCO})$, they are the $\left\{e_{r s}\right\}$ matrix and the labeled degree sequence $\left\{\vec{k}_{i}\right\}$ (which is omitted for the non-degree-corrected model, $\mathcal{H}=\mathrm{NDCO})$,

$$
\begin{aligned}
P\left(G \mid\left\{\vec{b}_{i}\right\}, \mathrm{DC}\right)= & \sum_{\left\{e_{r s}^{\prime}\right\},\left\{\vec{k}_{i}^{\prime}\right\}} P\left(G \mid\left\{\vec{b}_{i}\right\},\left\{e_{r s}^{\prime}\right\},\left\{\vec{k}_{i}^{\prime}\right\}\right) \\
& \times P\left(\left\{e_{r s}^{\prime}\right\}\right) P\left(\left\{\vec{k}_{i}^{\prime}\right\}\right) \\
= & P\left(G \mid\left\{\vec{b}_{i}\right\},\left\{e_{r s}\right\},\left\{\vec{k}_{i}\right\}\right) P\left(\left\{e_{r s}\right\}\right) P\left(\left\{\vec{k}_{i}\right\}\right),
\end{aligned}
$$

where the sum trivially contains only one term since, for the same graph $G$ and partition $\left\{\vec{b}_{i}\right\}$, there is only one possible choice for the $\left\{e_{r s}\right\}$ matrix and degree sequence $\left\{\vec{k}_{i}\right\}$ with nonzero probability, which is a convenient feature of the microcanonical model formulation considered here [the 
same holds for $\mathcal{H}=$ NDC, i.e., $P\left(G \mid\left\{\vec{b}_{i}\right\}\right.$, NDC $)=$ $\left.P\left(G \mid\left\{\vec{b}_{i}\right\},\left\{e_{r s}\right\}\right) P\left(\left\{e_{r s}\right\}\right)\right]$. Now if we want to compare two competing partitions $\left\{\vec{b}_{i}\right\}_{a}$ and $\left\{\vec{b}_{i}\right\}_{b}$, this can be done again via the posterior odds ratio $\Lambda$,

$$
\begin{aligned}
\Lambda & =\frac{P\left(\left\{\vec{b}_{i}\right\}_{a}, \mathcal{H}_{a} \mid G\right)}{P\left(\left\{\vec{b}_{i}\right\}_{b}, \mathcal{H}_{b} \mid G\right)} \\
& =\frac{P\left(G \mid\left\{\vec{b}_{i}\right\}_{a}, \mathcal{H}_{a}\right) P\left(\left\{\vec{b}_{i}\right\}_{a} \mid \mathcal{H}_{a}\right) P\left(\mathcal{H}_{a}\right)}{P\left(G \mid\left\{\vec{b}_{i}\right\}_{b}, \mathcal{H}_{b}\right) P\left(\left\{\vec{b}_{i}\right\}_{b} \mid \mathcal{H}_{b}\right) P\left(\mathcal{H}_{b}\right)} \\
& =\exp (-\Delta \Sigma),
\end{aligned}
$$

with $\Delta \Sigma=\Sigma_{a}-\Sigma_{b}$ being the difference in the description length, and in Eq. (28), it was assumed that $P\left(\mathcal{H}_{a}\right)=$ $P\left(\mathcal{H}_{b}\right)=1 / 2$, corresponding to a lack of $a$ priori preference for either model variant (which, in fact, makes $\Lambda$ identical to the Bayes factor [50]). This is a simple result, which enables us to use the difference in the description length directly in the computation of confidence levels. Being a ratio of probabilities, the value of $\Lambda$ has a straightforward interpretation: For a value of $\Lambda=1$, both models explain the data equally well, and for values of $\Lambda<1$, model $a$ is rejected in favor of $b$ with a confidence increasing as $\Lambda$ diminishes. In order to simplify its interpretation, the values of $\Lambda$ are usually divided into regions corresponding to a subjective assessment of the evidence strength. A common classification is as follows [50]: Values of $\Lambda$ in the intervals $\{[1,1 / 3]$, $[1 / 3,1 / 10],[1 / 10,1 / 30],[1 / 30,1 / 100],[1 / 100,0]\} \quad$ are considered to be very weak, substantial, strong, very strong, and decisive evidence supporting model $b$, respectively. In the following, when comparing different models, we will always put the preferred model in the denominator of Eq. (27) such that $\Lambda \leq 1$.

Using the posterior odds ratio $\Lambda$ is more practical than some alternative model selection approaches, such as likelihood ratios. As has been recently shown [29], the likelihood distribution for the stochastic block model does not follow a $\chi^{2}$ distribution asymptotically for sparse networks, and hence the calculation of a $p$ value must be done via an empirical computation of the likelihood distribution, which is computationally costly and prohibitively so for very large networks. In contrast, computing $\Lambda$ can be done easily, and it properly accounts for the increased complexity of models with larger parameters and protects against overfitting. However, it should be emphasized that these different model-selection approaches are designed to answer similar but not identical questions. Therefore, the most appropriate method should be the one that more closely matches the questions raised.

\section{EMPIRICAL NETWORKS}

The method outlined in the previous section allows one to determine the best model from the various available choices.
Here, we analyze some empirical examples, determine the most appropriate model, and examine the consequences of the balance struck between model complexity and quality of fit. We start with two small networks, the co-appearance of characters in the Victor Hugo novel "Les Misérables" [51], and a network of American college football games $[13,52]$. For both networks, we obtain the best partition according to all model variations and for a different number of groups $B$, and we compute the value of $\Lambda$ relative to the best model, as shown in Fig. 1. For the "Les Misérables" network, the best fit is a non-degree-corrected overlapping model that puts the most central characters in more than one group. All other partitions for different values of $B$ and model types result in values significantly below the plausibility line of $\Lambda=10^{-2}$, indicating that the overlapping model offers a better explanation for the data with a large degree of confidence. In particular, it offers a better description than the nonoverlapping model with degree correction. For the football network, on the other hand, the preferred model is nonoverlapping and without degree correction with $B=10$, which matches very well the assumed correct partition into 10 conferences. The groups are relatively homogeneous, with most nodes having similar degrees, such that degree correction becomes an extra burden, with very little added explanatory power. For this network, however, there are alternative fits with values of $\Lambda$ within the plausibility region, which means that the communities are not very strongly defined; they admit alternative partitions with $B=9$ and $B=8$ groups which cannot be confidently discarded given the evidence in the data.

Degree correction tends to become a better choice for larger data sets which display stronger degree variability. One example of this is the network of political blogs obtained by Adamic et al. [53]. For this network, the best model is a degree-corrected, overlapping partition into $B=7$ groups, shown in Fig. 2. Compared to this partition, the best alternative model without overlap divides the network into $B=12$ groups but has a posterior odds ratio significantly below the plausibility region. (In Ref. [7], using the same nonoverlapping model, a value of $B=15$ was found. This result is due to the difference in the description length for the degree sequence, where here we use a more complete estimation than in Ref. [7], which results in this slight difference.) It should be observed that the nonoverlapping version captures well the segregation into two groups (Republicans and Democrats) at the topmost level of the hierarchy. The overlapping version, on the other hand, tends to classify half-edges belonging to different camps into different groups, which is compatible with the accepted division, but the upper layers of the hierarchy do not reflect this, and it prefers to merge together groups that belong to different factions but that have otherwise similar roles in the topology.

Overlapping partitions, however, do not always provide better descriptions, even in situations where it might be 

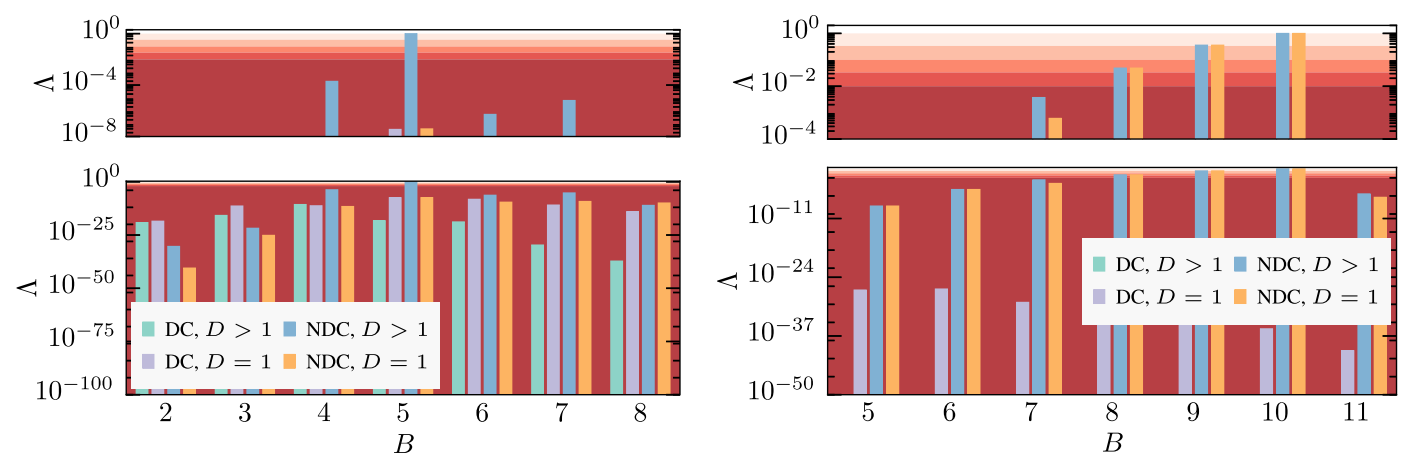

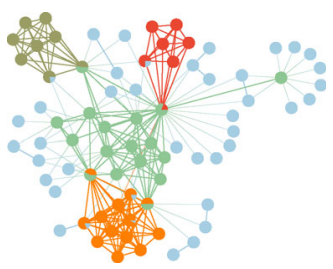

$B=5$,

non-degree-corrected, overlapping, $\Lambda=1$

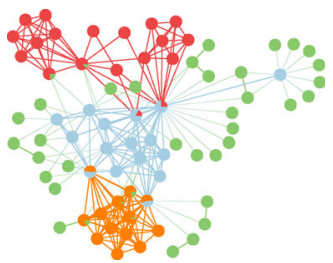

$B=4$,

non-degree-corrected, overlapping, $\Lambda \simeq 2 \times 10^{-4}$,

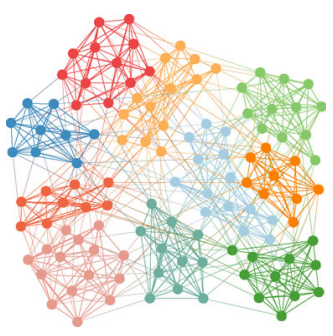

$B=10$,

non-degree-corrected, nonoverlapping, $\Lambda=1$

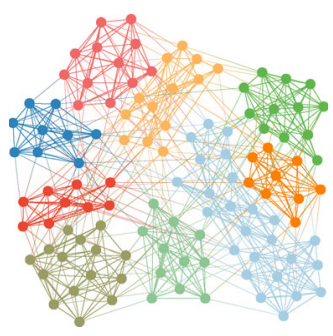

$B=9$,

non-degree-corrected, nonoverlapping, $\Lambda \simeq 0.36$

FIG. 1. Left: Values for posterior odds ratio $\Lambda$ for the network of co-appearances of characters in the novel "Les Misérables," for all model variations $(D>1$ indicates an overlapping model, "DC" a degree-corrected model, and "NDC" a non-degree-corrected one). The models with the best and second-best fits are shown at the bottom. Right: Same as in the left, but for the American college football network.

considered more intuitive. One of the contexts where overlapping communities are often considered to be better explanations is in social networks, where different social circles could be represented as different groups (e.g., family, co-workers, friends, etc.), and one could belong to more than one of these groups. This is illustrated well by so-called "ego networks," where one examines only the immediate neighbors of a node and their mutual connections. One such network, extracted from the Facebook online social network [55], is shown in Fig. 3. The common

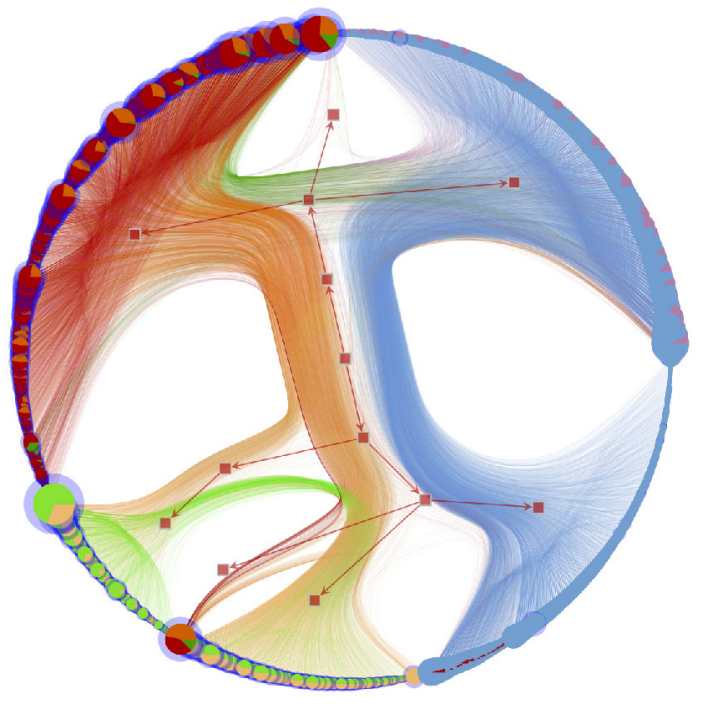

$B=7$, overlapping, degree-corrected, $\Lambda=1$

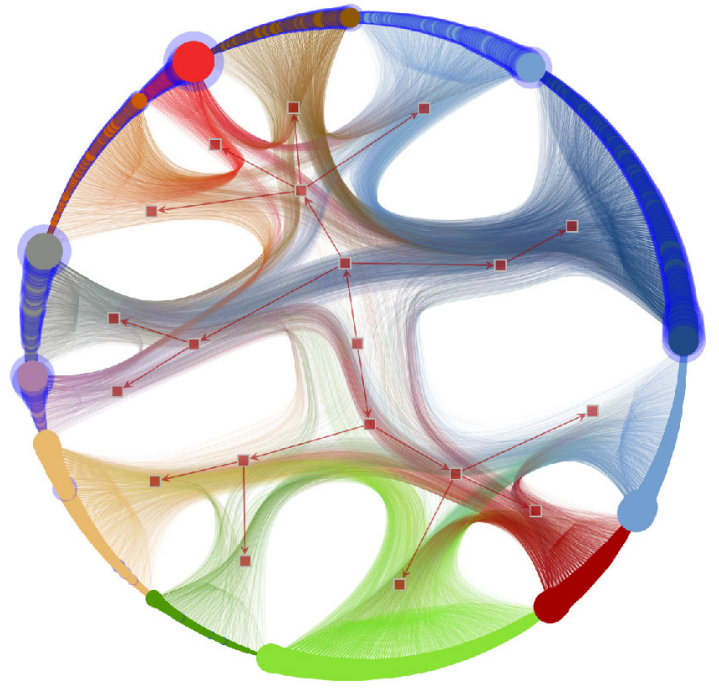

$B=12$, nonoverlapping, degree-corrected, $\log _{10} \Lambda \simeq-747$

FIG. 2. The network of political blogs by Adamic et al. [53]. The left panel shows the best model with an overlapping partition, and the right shows the best nonoverlapping one. Nodes with a blue halo belong to the Republican faction, as determined in Ref. [53]. For the visualization, the hierarchical edge-bundles algorithm [54] was used. 

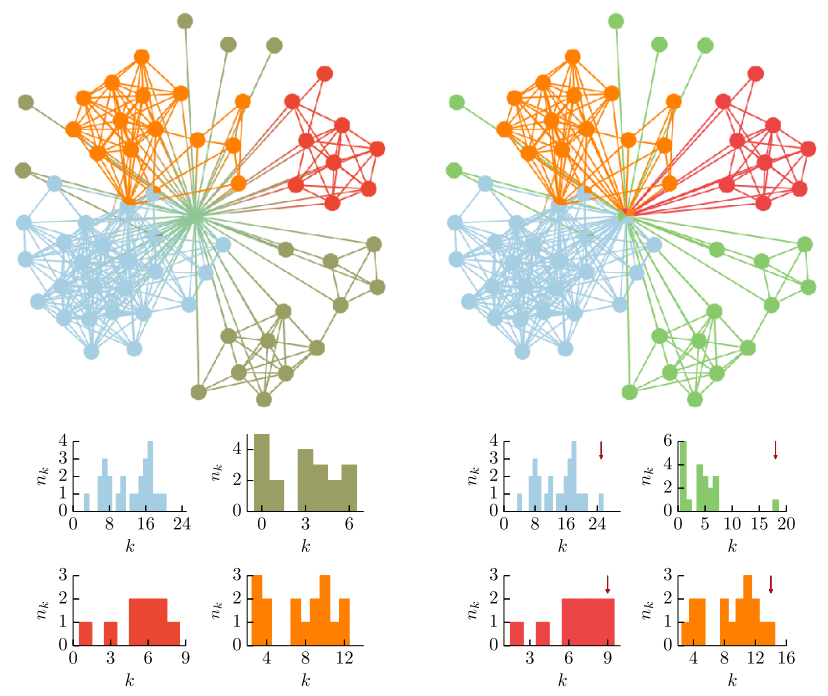

$B=5$, overlapping

non-degree-corrected, $\Lambda=1$

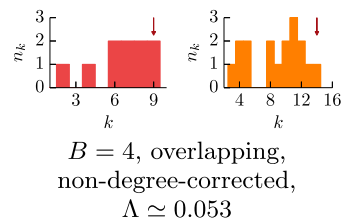

FIG. 3. Ego network of Facebook contacts [55]. Left: The best model fit across all model variations, which puts the ego node in its own group. Right: The alternative hypothesis where the node is split into several groups. Below each network are shown the degree distributions inside each group. The arrow marks the degree of the ego node.

interpretation of networks such as these is shown on the right in Fig. 3, and it corresponds to a partition of the central "ego" node so that it belongs to all of the different circles. Under this interpretation, the ego node is only special in the sense that it belongs to all groups, but inside each group, it is just a common member. However, among all model variants, the best fit turns out to be the one where the ego node is put separately in its own group, as shown in the left in Fig. 3. In this example, it is easy to see why this is the case: If we observe the degree distribution inside each group for the network on the left, we see that there is no strong degree variation. On the right, as the ego is included in each group, it becomes systematically the most connected node. This is simply by construction since the ego must connect to every other node. The only situation where the ego would not stand out inside each group would be if the communities were cliques. Hence, since the ego is not a typical member of any group, it is simpler to classify it separately in its own group, which is selected by the method as being a more plausible hypothesis. Note that degree correction is not selected as the most plausible solution since it is burdened with the individual description of every degree in the network, which is fairly uniform with the exception of the ego. One can imagine a different situation where there would be other very well connected nodes inside each group so that the ego could be described as a common member of each group, but this not observed in any other network obtained in Ref. [55]. Naturally, if one considers the complete network, of which the ego neighborhood is only a small part, the situation may change since there may be members of each group to which the ego does not have a direct connection.

When performing model selection for larger networks, it is often the case that the overlapping models are not chosen. In Table I, we show the results for many empirical networks belonging to different domains. For the majority of cases, the nonoverlapping degree-corrected models are selected. There are, however, many exceptions, which include two social networks (Gowalla and Brightkite [56]), the global airport network of openflights.com, the neuronal network of $C$. elegans [57], the political blog network already mentioned, the arXiv co-authorship networks [58] [in the fields of general relativity and quantum cosmology (gr-qc), high-energy physics (hep-th), condensed matter (condmat), and astronomy (astro-ph)], co-authorship in network science [59], and the network of genes implicated in diseases [60], for which some version of the overlapping model is chosen. Interestingly, for the arXiv co-authorship network in high-energy physics/phenomenology (hep-ph), a nonoverlapping model is selected instead. For only one of the remaining four arXiv networks (astro-ph), the degreecorrected version of the overlapping model is selected, whereas for the other three, the non-degree-corrected version is preferred. Hence, for co-authorship networks, the model selection procedure seems to correspond to the intuition that they are composed predominantly of overlapping groups [15].

We take the arXiv cond-mat network as a representative example of the differences between the inferred models. As can be seen in Fig. 4, although the degree distribution is very broad, the inferred labeled degree distribution is narrower, meaning that many large-degree nodes can be well explained as having a smaller degree of any single type, but belonging simultaneously to many groups (in the specific context of this network, prolific authors tend to be the ones that belong to many different types of collaborations). The distribution of mixture sizes $n_{d}$ almost always has a maximum at $d=1$, meaning that most nodes belong to one group, but with a tail that is comparatively broad (this seems to be a general feature that is observed in the majority of networks analyzed). The distribution of group sizes can be very different, depending on which model is used. Nonoverlapping models without degree correction tend to find groups that are strongly correlated with degrees [19] and hence lead to a broad distribution of group sizes when the degree distribution is also broad. On the other hand, both degree correction and group overlap tend to change the distribution considerably. In the literature, there are often claims of community sizes following power-law distributions $[21,78-80]$ with figures similar to the lowerleft panel of Fig. 5. Regardless of the validity of this hypothesis for the various methods used in the literature, this is certainly not the case for the overlapping model, as shown in the lower-right panel of the same figure. Indeed, 
TABLE I. Comparison of different models for many empirical networks. The columns in the table correspond to the data-set number (with the name given at the bottom of the table), the number of nodes $N$, the average degree $\langle k\rangle=2 E / N$, and the posterior odds ratios relative to the best model for the degree-corrected overlapping $\left(\Lambda_{\mathrm{DCO}}\right)$, the degree-corrected nonoverlapping $\left(\Lambda_{\mathrm{DC}}\right)$, non-degreecorrected overlapping $\left(\Lambda_{\mathrm{NDCO}}\right)$, and non-degree-corrected nonoverlapping $\left(\Lambda_{\mathrm{NDC}}\right)$ models. Missing entries correspond to situations where the best overlapping partition turns out to be nonoverlapping. The last three columns show some parameters of the best model: the number of groups $B$, the average mixture size $\langle d\rangle$, and the description length per edge (in bits per edge).

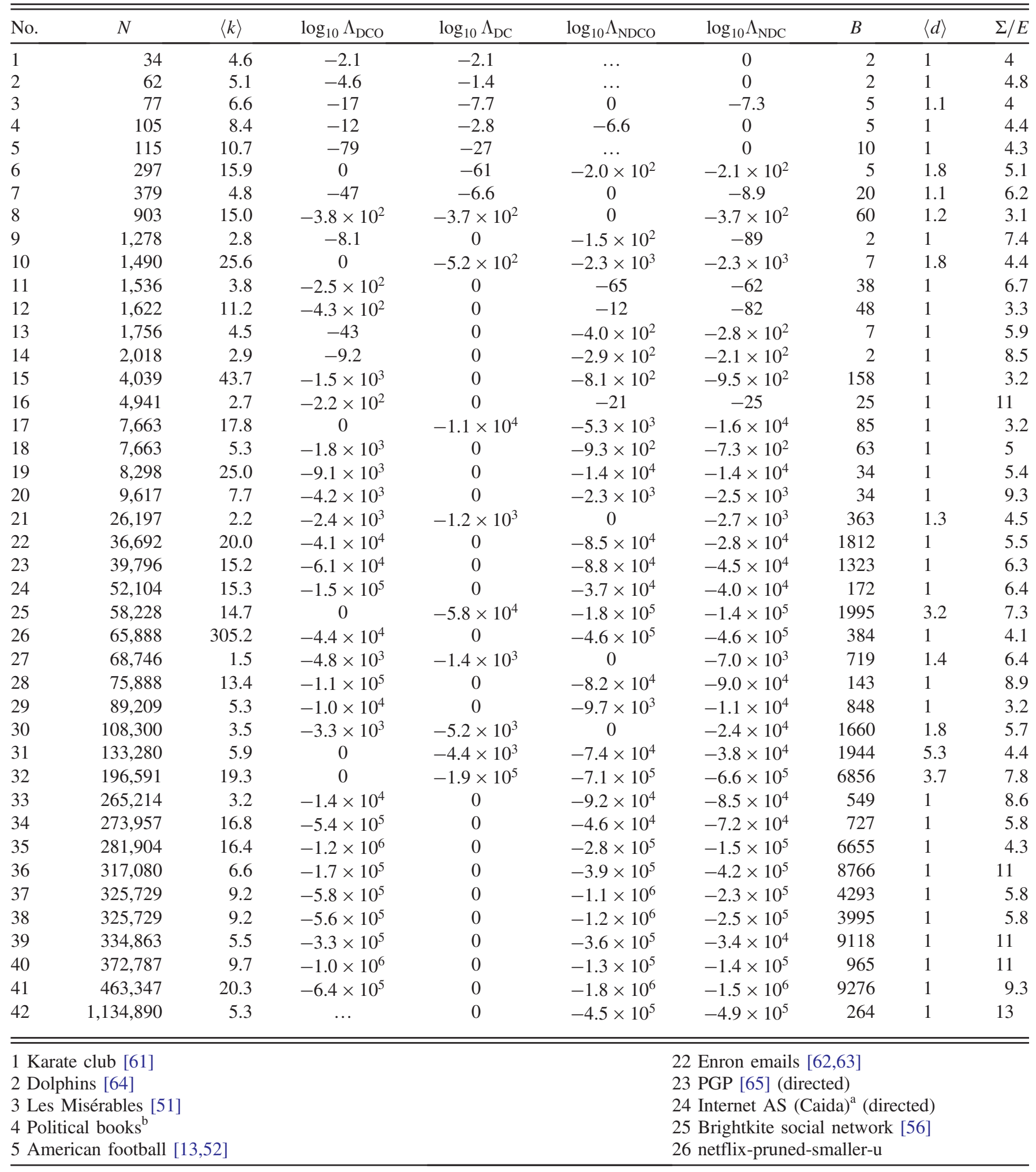


TABLE I. (Continued)

6 C. elegans neurons [57] (directed)

7 Co-authorships in network science [59]

8 Disease genes [60]

9 Yeast protein interactions (CCSB-YI11) [67]

10 Political blogs [53] (directed)

11 Yeast protein interactions (LC) [68]

12 Yeast protein interactions (combined AP/MS) [69]

13 E. coli gene regulation [70] (directed)

14 Yeast protein interactions (Y2H union) [67]

15 Facebook egos [55]

16 Power grid [57]

17 Airport routes ${ }^{\mathrm{c}}$ (directed)

18 Airport routes

19 Wikipedia votes $[75,76]$ (directed)

20 Human protein interactions (HPRD r9) [77]

21 arXiv co-authors (gr-qc) [58]
27 arXiv co-authors (hep-th) [58]

28 Epinions.com trust network [66] (directed)

29 arXiv co-authors (hep-ph) [58]

30 arXiv co-authors (cond-mat) [58]

31 arXiv co-authors (astro-ph) [58]

32 Gowalla social network [56]

33 EU email [58] (directed)

34 Flickr [71]

35 Web graph of stanford.edu. [72] (directed)

36 DBLP Collaboration [73]

37 Web graph of nd.edu. [72] (directed)

38 WWW [74] (directed)

39 Amazon product network [73]

40 IMDB film actor $^{\mathrm{d}}[48]$

41 APS citations ${ }^{\mathrm{e}}$ (directed)

42 Youtube social network [73]

${ }^{\mathrm{a}}$ Retrieved from http://www.caida.org.

${ }^{\mathrm{b}} \mathrm{V}$. Krebs, retrieved from http://www-personal.umich.edu/mejn/netdata.

${ }^{c}$ Retrieved from http://openflights.org.

${ }^{\mathrm{d}}$ Retrieved from http://www.imdb.com/interfaces.

${ }^{\mathrm{e}}$ Retrieved from http://publish.aps.org/dataset.

for most networks analyzed, the model that best fits the data (which tends to be degree-corrected and nonoverlapping) shows no vestige of group sizes following a scale-free distribution. Some further examples of this are shown in Fig. 5, where characteristic size scales can be clearly identified.
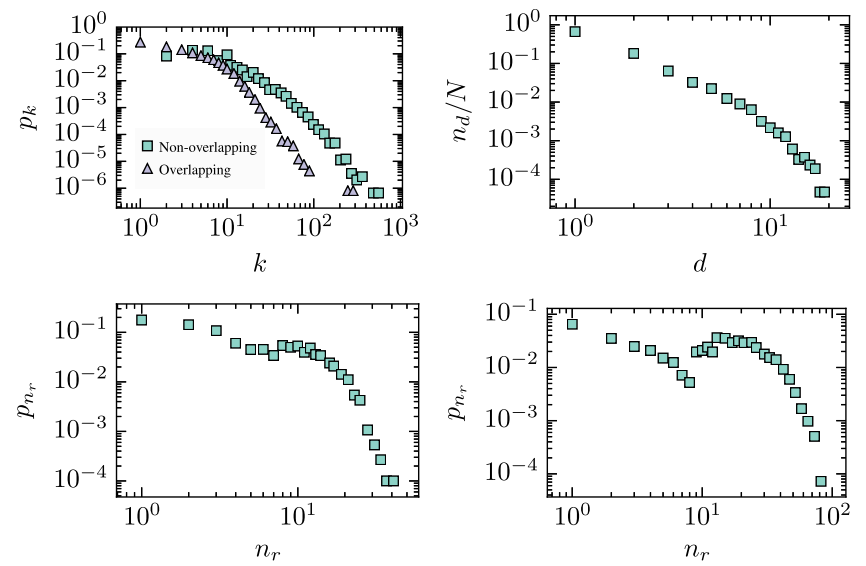

FIG. 4. Statistical properties of the best model inferred for the network of arXiv co-authors in the field of condensed matter (cond-mat). Top-left panel: Degree distribution of the original network and of the overlapping model (where the labeled degree sequence $\left\{\vec{k}_{i}\right\}$ is flattened into a single histogram for all labeled degrees $\left\{k_{i}^{r}\right\}$ ). Top-right panel: Distribution of mixture sizes, $n_{d}$. Bottom-left panel: Distribution of group sizes for the best-fitting nonoverlapping, non-degree-corrected model. Bottom-right panel: Distribution of group sizes for the best-fitting overlapping, non-degree-corrected model.

\section{MODEL IDENTIFIABILITY: OVERLAPPING VS NONOVERLAPPING}

A central issue when selecting between nonoverlapping and overlapping models is to decide when a group of nodes should belong simultaneously to two or more groups, or if these nodes should be better represented by a single membership to a different unique group. The choice is not always immediately obvious since we can always generate very similar networks with either model. If we generate a network with the overlapping model but treat it
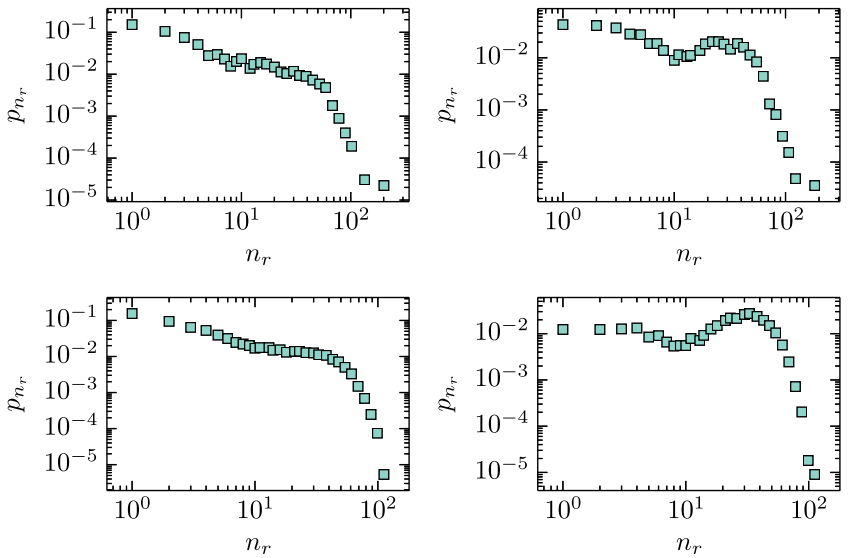

FIG. 5. Distribution of group sizes for the best-fitting nondegree-corrected nonoverlapping model (left) and the degreecorrected nonoverlapping model (right), for the PGP [65] (top) and DBLP collaborations [73] (bottom) networks. In both cases, the degree-corrected model provides a better fit, as shown in Table I. 
as if it were generated by the nonoverlapping model, with each distinct mixture $\vec{b}$ corresponding to a separate nonoverlapping group, the associated entropy will be

$$
\mathcal{S}_{t}^{\prime} \simeq E-\frac{1}{2} \sum_{\vec{b}_{1} \vec{b}_{2}} e_{\vec{b}_{1} \vec{b}_{2}} \ln \left(\frac{e_{\vec{b}_{1} \vec{b}_{2}}}{n_{\vec{b}_{1}} n_{\vec{b}_{2}}}\right),
$$

where

$$
e_{\vec{b}_{1} \vec{b}_{2}}=\sum_{r s} b_{1}^{r} b_{2}^{s} \frac{e_{r s}}{n_{r} n_{s}} n_{\vec{b}_{1}} n_{\vec{b}_{2}}
$$

is the expected number of edges between mixtures $\vec{b}_{1}$ and $\vec{b}_{2}$. By exchanging the sums and using Jensen's inequality, we observe directly that

$$
\mathcal{S}_{t}^{\prime} \leq E-\frac{1}{2} \sum_{r s} e_{r s} \ln \left(\frac{e_{r s}}{n_{r} n_{s}}\right),
$$

with the right-hand side being the entropy of the original overlapping model $\mathcal{S}_{t}$ and with the equality holding only if the original model happens to be nonoverlapping to begin with. Thus, the nonoverlapping model will invariably possess a lower entropy. Nevertheless, the overlapping hypothesis may still be preferred if the number of groups $B$ is sufficiently smaller than the number of individual $\vec{b}$ mixtures so that the total description length is shorter. It should be observed, however, that since one model is contained inside the other, the difference in the description length can be interpreted simply as the difference in the prior probabilities for the model parameters. As the amount of available data increases, the effect of the priors should "wash out," and the description length should be increasingly dominated by the model entropy alone. In these cases, one should expect the nonoverlapping model to be preferred, regardless of the specific model that was used to generate the data. However, differently from models that generate independent data points, the "amount of available data" for network models is a finer issue. In the case of the stochastic block model, it involves the simultaneous scaling of the number of edges $E$, the number of nodes $N$, and the number of groups $B$.

As a case example, here we consider a simple overlapping assortative model, with $e_{r s}=$ $2 E\left[\delta_{r s} c / B+\left(1-\delta_{r s}\right)(1-c) / B(B-1)\right]$, with $c \in[0,1]$ controlling the degree of assortativity. The mixtures are parametrized as $n_{\vec{b}}=C \prod_{r} \mu^{b_{r}}$, with $C$ being a normalization constant, and $\mu \in[0,1]$ controlling the degree of overlap. For $\mu \rightarrow 0$, we obtain asymptotically a nonoverlapping partition with $n_{r}=N / B$, and for $\mu=1$, all mixtures $\vec{b}$ have the same size. We compare the difference in description length between this model and its equivalent parametrization with each mixture as a separate group.
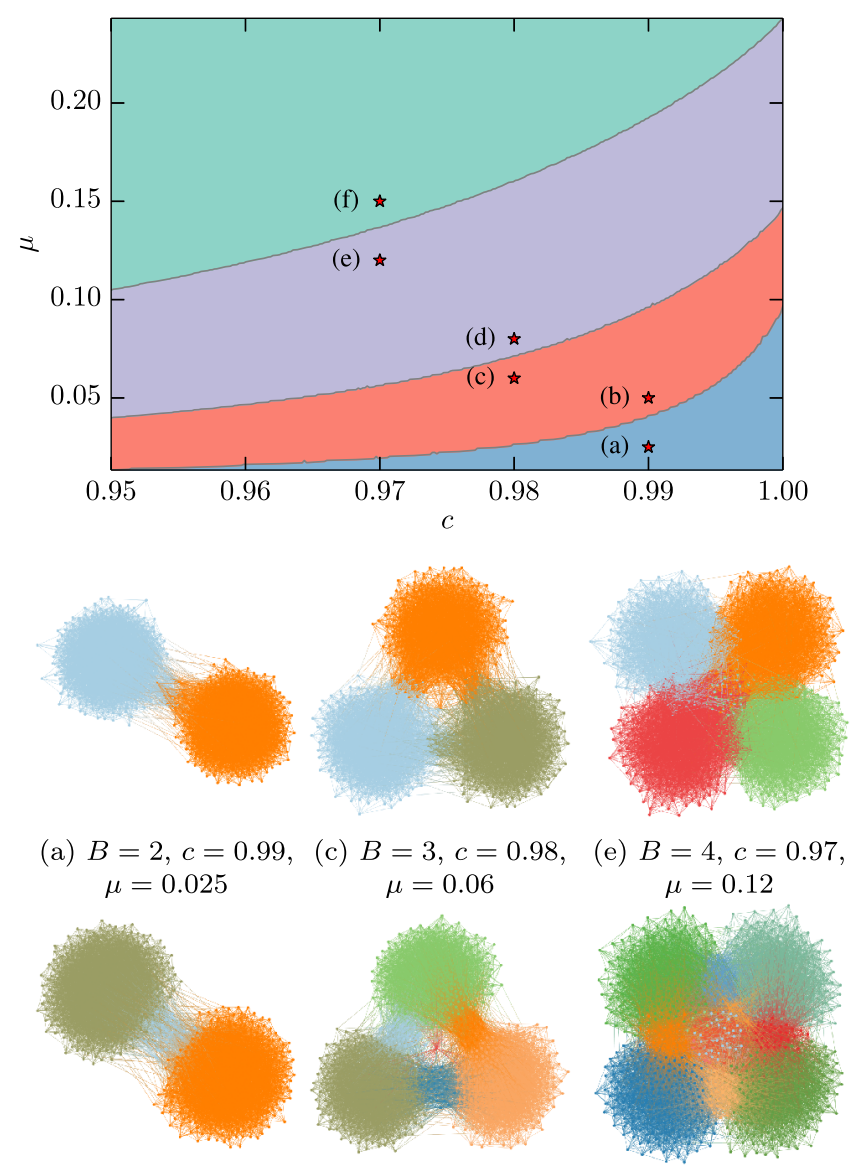
(b) $B=3, c=0.99$, $\mu=0.05$

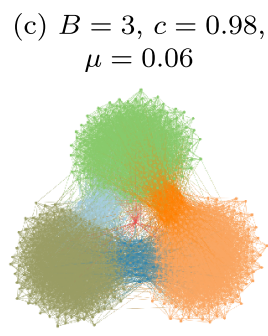

(e) $B=4, c=0.97$,

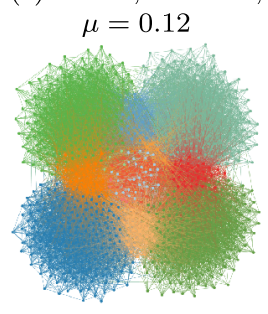

(d) $B=7, c=0.98$, $\mu=0.08$

(f) $B=15, c=0.97$, $\mu=0.15$

FIG. 6. Top: Parameter regions for the model considered in the main text, with $N=10^{3}$ and $\langle k\rangle=2 E / N=20$. Each curve corresponds to one value of $B$ and separates a region above where the nonoverlapping model is preferred from a region below where the overlapping model is chosen. Bottom: Networks and their preferred partitions, corresponding to parameter values indicated in the top panel.

As can be seen in Fig. 6, for any given value of $c$, there is a value of $\mu$ above which the nonoverlapping model is preferred. In this parameter region, the group intersections are sufficiently well populated with nodes, so their representation as individual groups is chosen. For values of $\mu$ below this value, the intersections are significantly smaller than the nonoverlapping portion. In this case, the data are better explained as larger groups of almost nonoverlapping nodes, with few nodes at the intersections. The boundary separating the two regions recedes upwards as the number of groups $B$ is increased, meaning that a larger number of distinct intersections can compensate for a smaller number of nonoverlapping nodes. It should also be pointed out that the boundaries move downwards as the number of nodes and edges is increased, such that the average degree in the network remains the same (not shown), so it is not only the relative sizes of the intersections that are the relevant 
properties but also their absolute sizes. The same occurs if the average degree increases and everything else remains constant. Hence, in the limit of sufficient data, either with the number of nodes inside each group and intersections becoming sufficiently large, or with each part becoming sufficiently dense, the nonoverlapping model is the one that will be selected. For empirical networks, this may not be the most representative scaling scenario since the most appropriate number of groups and degree of overlap may in fact follow any arbitrary scaling, and hence, the overlapping model may still be selected, even for very large or very dense networks. Nevertheless, this example seems to suggest that the nonoverlapping model is general enough to accommodate structures generated by the overlapping model in these limiting cases and may serve as a partial explanation as to why the overlapping model is seldom selected in the empirical systems analyzed in Sec. IV.

\section{INFERENCE ALGORITHM}

The inference procedure consists in finding the labeling of the half-edges of the graph such that the description length is minimized. Such global optimization problems are often NP hard and require heuristics to be solvable in practical time. One possibility is to use the Markov chain Monte Carlo (MCMC) method, which consists in modifying the block membership of each half-edge in a random fashion, and accepting or rejecting each move with a probability given as a function of the description length difference $\Delta \Sigma$. By choosing the acceptance probabilities in the appropriate manner, i.e., by enforcing ergodicity and detailed balance, one can guarantee that the labelings will be sampled with the correct probability after a sufficiently long equilibration time is reached. However, naive formulations of the Markov chain will lead to very long equilibration times, which become unpractical for large networks. Here, we adapt the algorithm developed in Ref. [81] for the nonoverlapping case which implements a fast Markov chain. It consists in the move proposal of each half-edge incident on node $i$ of type $r$ to type $s$ with a probability given by

$$
p(r \rightarrow s \mid t)=\frac{e_{t s}+\epsilon}{e_{t}+\epsilon B},
$$

where $t$ is the block labeling the half-edge opposing a randomly chosen half-edge incident to the same node as the half-edge being moved, and $\epsilon \geq 0$ is a free parameter. Equation (32) shows that we attempt to guess the label of a given half-edge by inspecting the group membership of the neighbors of the node to which it belongs and using the currently inferred model parameters to choose the most likely group to which it should be moved. It should be emphasized that this move proposal does not result in a preference for either assortative or dissortative networks since it depends only on the matrix $\left\{e_{r s}\right\}$ currently inferred.
For any choice of $\epsilon>0$, this move proposal preserves ergodicity but not detailed balance. This last characteristic can be enforced via the Metropolis-Hastings criterion $[82,83]$ by accepting each move with a probability $a$ given by

$$
a=\min \left\{e^{-\beta \Delta \Sigma} \frac{\sum_{t} p_{t}^{i} p(s \rightarrow r \mid t)}{\sum_{t} p_{t}^{i} p(r \rightarrow s \mid t)}, 1\right\},
$$

where $p_{t}^{i}$ is the fraction of opposing half-edges of node $i$ which belong to block $t$, and $p(s \rightarrow r \mid t)$ is computed after the proposed $r \rightarrow s$ move (i.e., with the new values of $e_{t r}$ ), whereas $p(r \rightarrow s \mid t)$ is computed before. The parameter $\beta$ in Eq. (33) is an inverse temperature, which can be used to sample partitions according to their description length $(\beta=1)$ or to find the ground state $(\beta \rightarrow \infty)$. As explained in Ref. [81], this move proposal, as well as the computation of $a$, can be done efficiently, with minimal book-keeping, so that a sweep of the network (where each half-edge move is attempted once) is done in time $O(E)$, independent of the number of groups $B$. This is true even in the overlapping case since updating Eqs. (1), (2), (6) and (15) after each half-edge move can be done in time $O(1)$.

As discussed in Ref. [81], although the MCMC method above succeeds in equilibrating faster than a naive Markov chain, it still suffers from a strong dependence on how close one starts from the global minimum. Usually, starting from a random partition of the half-edges leads to metastable states where the Markov chain seems to have equilibrated, but in fact, the network structure has only been partially discovered and will move from such configurations only after a very long time. This problem is common to many inference procedures based on local moves such as expectation maximization [20] and belief propagation $[84,85]$. In Ref. [81], a multilevel agglomerative heuristic was proposed, which significantly alleviates this problem. It consists in equilibrating the chain for a larger number of groups and then merging the groups using the same algorithm used for the block membership moves. This method, however, cannot be used unmodified in the overlapping case since the strict merging of groups will not properly explore the landscape of possible overlapping partitions. We therefore modify the approach as follows. Before groups are merged, the half-edges belonging to each one of them are split into subgroups corresponding to the different group memberships at the opposing sides. These subgroups are then treated as separate groups and are merged together until the desired number of groups is achieved. All the details of the algorithm beyond this modification are performed exactly as described in Ref. [81]. Since this algorithm usually does a good job in finding a partition very close to the final one, it also tends to perform very well when the algorithm is turned into a greedy heuristic, by starting with $B=2 E$ and each half-edge in its own group and by making $\beta \rightarrow \infty$. An example of a typical outcome of the greedy algorithm is 

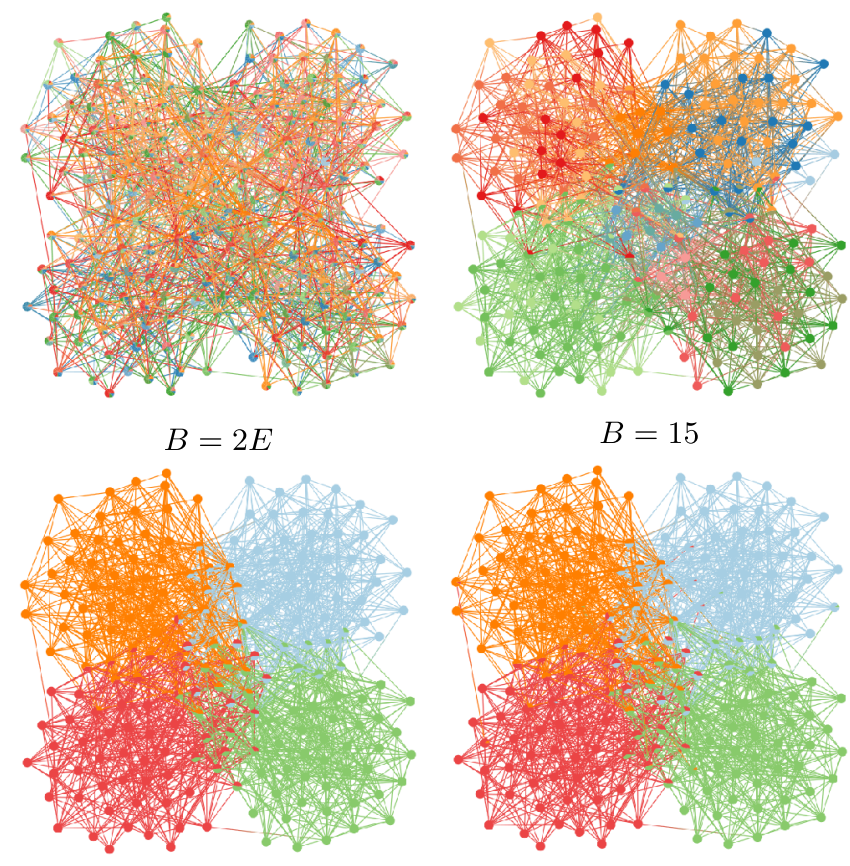

$B=4$

$B=4$, planted

FIG. 7. Typical outcome of the greedy multilevel agglomerative algorithm described in the text, for a network sampled from the overlapping model with $B=4$. The different panels show the progression of the algorithm from $B=2 E$ to $B=4$. The panel on the lower right shows the planted partition used to generate the network.

shown in Fig. 7. The greedy version is very fast, with an overall complexity of $O\left(E \ln ^{2} E\right)$, which makes it usable for very large networks. Note that this complexity is independent of the number of groups, $B$. This is in strong contrast to other methods proposed for the same problem, such as the stochastic optimization algorithm of Gopalan et al. [86] and the expectation maximization algorithm of Ball et al. [20], both of which have a complexity of $O(E B)$ per sweep, although they only consider strictly assortative models, and applying the same techniques to the more general models considered here would lead to an $O\left(E B^{2}\right)$ complexity, similar to belief propagation algorithms for nonoverlapping models $[29,85]$. Although these approaches can be very efficient if the number of groups is very small, they quickly become prohibitive if the most appropriate number of groups scales as some function of the system size (which seems to generally be the case when model selection is applied; see Table I and Ref. [7]), which is not an issue with the algorithm described above. It should also be noted that none of the other algorithms mentioned [20,29,85,86] is designed to overcome metastable solutions, like the multilevel approach presented here.

For most networks analyzed in this work, the fast heuristic version of the algorithm was used, together with the algorithm described in Ref. [7], to infer the upper layers of the hierarchy (which includes the determination of the number of groups $B$ at the lowest level, in addition to the entire hierarchy, in a nonparametric fashion). (A complete implementation of the algorithm is freely available as part of the graph-tool library [87] at http://graph-tool .skewed.de.)

\section{CONCLUSION}

We presented a method of inferring overlapping and degree-corrected versions of the stochastic block model based on the MDL that avoids overfitting and allows for the comparison between model classes. Based on a Bayesian interpretation of MDL, we derived a posterior odds ratio test that yields a degree of confidence with which models can be selected or discarded. In applying this method to a variety of empirical networks, we obtained that, for the majority of them, the nonoverlapping degree-corrected model variant is the one that best fits the data.

The relative success of the degree-corrected model implies that intrinsic node propensities are an important aspect of the network formation of many systems, which are not sufficiently well described by the sole division into node classes. We note, however, that there are exceptions to this, as there are a few networks that do not show enough statistical evidence to justify the additional parameters of the degree-corrected model. In these networks, the groups themselves seem to be the leading descriptors of the network structure, with the degree sequence itself providing little additional explanatory power.

Although overlapping structures are often considered to be more intuitive explanations for some networks, we showed that in many representative cases, the nonoverlapping model can accommodate the same structure while providing a more parsimonious description of the data. This contradicts results obtained with nonstatistical methods $[14,15]$, which claimed that many or even most networks are better described by overlapping groups. We believe that this conclusion is most likely a result of overfitting: Since there are more overlapping structures than nonoverlapping ones, it is easier to find them in the data. We expect this fact to bear on tasks that require high-quality fits, such as the prediction of missing or spurious links $[6,8]$, or other generalizations of the data.

The models considered in this work generate unlabeled networks, without any other properties associated with the nodes or edges. However, it is often the case that either the nodes or edges have weights $[25,40,88]$ or are of different types [24,27], or have temporal information [28]. This sort of additional data may corroborate the evidence supporting the generation via a specific type of model (e.g., with overlaps) and tip the scale towards it. Therefore, the results presented in this paper should not be interpreted as a statement on the suitability of the abstract notion of overlapping structures in general, only of the specific formulations considered. However, the approach presented here is generalizable to these other cases as well, by 
augmenting the model to generate covariates associated with the edges and nodes [25]. Furthermore, one should be able to perform a similar comparison with models which belong to very different classes, such as latent space [89] models, or others.

\section{ACKNOWLEDGMENTS}

This work was funded by the University of Bremen, under the funding program $\mathrm{ZF} 04$.

\section{APPENDIX A: DIRECTED GRAPHS}

The same approach of the main text can be carried over to directed graphs with no difficulties. In this case, the edge counts are, in general, asymmetric, $e_{r s} \neq e_{s r}$, which leads to the entropy for the non-degree-corrected model [42],

$$
\mathcal{S}_{t} \simeq E-\sum_{r s} e_{r s} \ln \left(\frac{e_{r s}}{n_{r} n_{s}}\right) .
$$

For the degree-corrected case, there are two degree sequences for the labeled out- and in-degrees, $\left\{k_{i}^{+r}\right\}$ and $\left\{k_{i}^{-r}\right\}$, respectively. Applying the same argument as for the undirected case, the entropy becomes [42]

$\mathcal{S}_{d} \simeq-E-\sum_{r s} e_{r s} \ln \left(\frac{e_{r s}}{e_{r}^{+} e_{s}^{-}}\right)-\sum_{i r} \ln k_{i}^{+r} !-\sum_{i r} \ln k_{i}^{-r} !$,

where $e_{r}^{+}=\sum_{s} e_{r s}$ and $e_{r}^{-}=\sum_{s} e_{s r}$.

The description length for the overlapping partition is identical to the undirected case, with $\mathcal{L}_{p}$ given by Eq. (6). For the labeled degree sequence, we have instead

$$
\begin{aligned}
\mathcal{L}_{\kappa}= & \sum_{r} \ln \left(\left(\begin{array}{c}
m_{r} \\
e_{r}^{+}
\end{array}\right)\right)+\ln \left(\left(\begin{array}{c}
m_{r} \\
e_{r}^{-}
\end{array}\right)\right) \\
& +\sum_{\vec{b}} \min \left(\mathcal{L}_{\vec{b}}^{(1)}, \mathcal{L}_{\vec{b}}^{(2)}\right),
\end{aligned}
$$

with

$$
\mathcal{L}_{\vec{b}}^{(1)}=\sum_{r} \ln \left(\left(\begin{array}{c}
n_{\vec{b}} \\
e^{+r} \\
\vec{b}
\end{array}\right)\right)+\ln \left(\left(\begin{array}{c}
n_{\vec{b}} \\
e_{\vec{b}}^{-r}
\end{array}\right)\right)
$$

and

$\mathcal{L}_{\vec{b}}^{(2)}=\sum_{r} b_{r}\left(\ln \Xi_{\vec{b}}^{r+}+\ln \Xi_{\vec{b}}^{r-}\right)+\ln n_{\vec{b}} !-\sum_{\vec{k}} \ln n_{\vec{k}^{+}, \vec{k}^{-}}^{\vec{b}^{-}} !$,

where $\ln \Xi_{\vec{b}}^{r+}$ and $\ln \Xi_{\vec{b}}^{r-}$ are computed as in Eq. (12) but using $e_{\vec{b}}^{+r}=\sum_{\vec{k}^{+}, \vec{k}^{-}} k_{r}^{+} n_{\vec{k}^{+}, \vec{k}^{-}}^{\vec{b}}$ and $e_{\vec{b}}^{r-}=\sum_{\vec{k}^{+}, \vec{k}^{-}} k_{r}^{-} n_{\vec{k}^{+}, \vec{k}^{-}}^{\vec{b}}$, respectively, which give the total number of out and in edges incident on the mixture $\vec{b}$. In the previous equations, the counts $n_{\vec{k}^{+}, \vec{k}^{-}}^{\vec{b}}$ refer to the joint distribution of labeled in and out degrees, so each vector $\vec{k}^{+/-}$describes the in and out degrees labeled according to degree membership, i.e., $\vec{k}_{i}^{+}=\left\{k_{i}^{+r}\right\}$ and $\vec{k}_{i}^{-}=\left\{k_{i}^{-r}\right\}$.

\section{APPENDIX B: POISSON MODELS}

\section{Non-degree-corrected}

This approximation of the formulation with "hard" constraints of the multiple membership model discussed in the main text is closely related to a Poisson variant of the model with "soft" constraints, where each half-edge of the graph is labeled with a latent variable specifying which group memberships were responsible for its existence, and the number of edges of type $(r, s)$ between nodes $i$ and $j, A_{i j}^{r s}$, is independently sampled according to a Poisson distribution (similar to Refs. [19,20]), so the likelihood becomes

$$
P\left(G \mid\left\{\vec{b}_{i}\right\},\left\{p_{r s}\right\}\right)=\prod_{i>j} \prod_{r \geq s} p_{r s}^{A_{i j}^{r s}} e^{-p_{r s} b_{i}^{r} b_{j}^{s}} / A_{i j}^{r s} !,
$$

where $p_{r s}$ is the average number of edges of type $(r, s)$ between nodes that belong to each group. The loglikelihood can be written as

$\ln P=\frac{1}{2} \sum_{r s} e_{r s} \ln p_{r s}-n_{r} n_{s} p_{r s}-\sum_{i>j} \sum_{r \geq s} \ln A_{i j}^{r s !} !$

Maximizing $\ln P$ with respect to $p_{r s}$, we obtain $\hat{p}_{r s}=e_{r s} / n_{r} n_{s}$, and hence

$\ln \hat{P}=-E+\frac{1}{2} \sum_{r s} e_{r s} \ln \left(\frac{e_{r s}}{n_{r} n_{s}}\right)-\sum_{i>j} \sum_{r \geq s} \ln A_{i j}^{r s} !$.

For simple graphs with $A_{i j}^{r s} \in\{0,1\}$, the last term in the above equation is equal to zero, and we have that the approximation of the likelihood of the model with "hard" constraints in the sparse case is identical to the exact maximum likelihood of the Poisson model with "soft" constraints.

This model is similar to the popular mixed membership stochastic block model (MMSBM) [18]; however, it differs in the important aspect that it generates strictly denser overlaps. In the MMSBM, the existence of an edge $A_{i j}$ is sampled from a Bernoulli distribution with parameter $\lambda_{i j}=\sum_{r s} \theta_{i}^{r} \theta_{j}^{s} p_{r s}$, where $\theta_{i}^{r}$ is the probability that node $i$ belongs to group $r$, such that $\sum_{r} \theta_{i}^{r}=1$, and $p_{r s} \in[0,1]$ is the probability that two nodes belonging to groups $r$ and $s$ are connected. Although for sparse graphs the differences between Poisson and Bernoulli models tend to disappear, with this parametrization, the density of the overlaps is mixed with normalized weights. More specifically, for a node $i$ which belongs simultaneously to groups $r$ and $s$, its 
expected degree is equal to the weighted average of the unmixed degrees, $\langle k\rangle_{i}=\theta_{i}^{r}\langle k\rangle_{r}+\theta_{i}^{s}\langle k\rangle_{s}$, where $\langle k\rangle_{r}=$ $\sum_{s} p_{r s} \sum_{i} \theta_{i}^{s}$ is the expected degree of a node that belongs only to group $r$. Thus, in the MMSBM, the nodes in the mixture have an intermediate density between the sparser and the denser groups. In contrast, in the model considered in the main text, as well as the Poisson model above, we have simply $\langle k\rangle_{i}=\langle k\rangle_{r}+\langle k\rangle_{s}$, and therefore, the overlaps are always strictly denser than the pure groups. In this respect, it is equivalent to other formulations of the MMSBM; see, e.g., Refs. [90,91].

\section{Degree-corrected}

A connection to a version of the model with "soft" constraints can also be made. We may consider each labeled entry $A_{i j}^{r s}$ in the adjacency matrix to be Poisson distributed with an average given by $\theta_{i}^{r} \theta_{j}^{s} \lambda_{r s}$,

$$
P\left(G \mid\left\{\vec{b}_{i}\right\},\left\{\lambda_{r s}\right\},\left\{\theta_{r}\right\}\right)=\prod_{i>j} \prod_{r \geq s}\left(\theta_{i}^{r} \theta_{j}^{s} \lambda_{r s}\right)^{A_{i j}^{r s}} e^{-\theta_{i}^{r} \theta_{j}^{s} \lambda_{r s}} / A_{i j}^{r s} !,
$$

where $A_{i j}^{r s}$ is the number of edges of type $(r, s)$ between nodes $i$ and $j$, and $\theta_{i}^{r}$ is the propensity with which a node receives an edge of type $r$. The log-likelihood can be written as

$$
\begin{aligned}
\ln P= & \frac{1}{2} \sum_{r s} e_{r s} \ln \lambda_{r s}+\sum_{i r} k_{i}^{r} \ln \theta_{i}^{r} \\
& -\sum_{r \geq s} \lambda_{r s} \sum_{i>j} \theta_{i}^{r} \theta_{j}^{s}-\sum_{i>j} \sum_{r \geq s} \ln A_{i j}^{r s} ! .
\end{aligned}
$$

Maximizing $\ln P$ with respect to $\left\{\lambda_{r s}\right\}$ and $\left\{\theta_{i}^{r}\right\}$, we obtain $\hat{\lambda}_{r s}=e_{r s} / e_{r} e_{s}$ and $\hat{\theta}_{i}^{r}=k_{i}^{r}$, and hence

$$
\begin{aligned}
\ln \hat{P}= & -E+\frac{1}{2} \sum_{r s} e_{r s} \ln \left(\frac{e_{r s}}{e_{r} e_{s}}\right)+\sum_{i r} k_{i}^{r} \ln k_{i}^{r} \\
& -\sum_{i>j} \sum_{r \geq s} \ln A_{i j}^{r s} ! .
\end{aligned}
$$

Again, for simple graphs with $A_{i j}^{r s} \in\{0,1\}$, the last term in the above equation is equal to zero; however, even in that case, the likelihood is not identical to the version with "hard" constraints considered above, as is the case for the single membership version as well [42]. Both likelihoods only become the same in the limit $k_{i}^{r} \gg 1$ such that $\ln k_{i}^{r} ! \simeq k_{i}^{r} \ln k_{i}^{r}-k_{i}^{r}$. Nevertheless, for the purpose of this paper, the differences between these models can be overlooked.

There is a direct connection between this model and the one proposed by Ball et al. [20]. In the not strictly assortative version of their model, the number of edges $A_{i j}$ is distributed according to a Poisson with average $\lambda_{i j}=\sum_{r s} \eta_{i}^{r} \eta_{j}^{s} \omega_{r s}$, where $\eta_{i}^{r}$ is the propensity with which node $i$ receives edges of type $r$ and $\omega_{r s}$ regulates the number of edges across groups. The total likelihood of that model is

$$
P\left(G \mid\left\{\vec{b}_{i}\right\},\left\{\omega_{r s}\right\},\left\{\eta_{r}\right\}\right)=\prod_{i>j} \lambda_{i j}^{A_{i j}} e^{-\lambda_{i j}} / A_{i j} !
$$

Since the sum of independent Poisson random variables is also distributed according to a Poisson, if we generate a graph with the model of Eq. (B4) and observe only the total unlabeled edge counts $A_{i j}=\sum_{r s} A_{i j}^{r s}$, they are distributed exactly like Eq. (B7), for the same choice of parameters $\theta_{i}^{r}=\eta_{i}^{r}$ and $\lambda_{r s}=\omega_{r s}$. Hence, the model of the main text is an equivalent formulation of the one in Ref. [20], where one keeps track of the latent variables specifying the exact type of each half-edge, instead of their marginal probability. This has the advantage that the maximum likelihood estimates for the model parameters $\lambda_{r s}$ and $\theta_{i}^{r}$ can be obtained directly by differentiation and do not require iterations of an expectation-maximization algorithm as in Ref. [20]. On the other hand, we are left with the determination of labels in the halfedges, which is done with the method already described in Sec. VI.

\section{APPENDIX C: MAXIMUM-ENTROPY ENSEMBLE OF COUNTS WITH CONSTRAINED AVERAGE}

Suppose we want to compute the number of all possible non-negative integer counts $\left\{n_{k}\right\}$, subject to a normalization constraint $\sum_{k=0}^{\infty} n_{k}=N$ and a fixed average $\sum_{k=0}^{\infty} k n_{k}=E$. This number can be obtained approximately by relaxing the constraints so that they hold only on average. The maximum entropy ensemble given these constraints is the one with the probabilities $P\left(\left\{n_{k}\right\}\right)=$ $e^{-H\left(\left\{n_{k}\right\}\right)} / Z$, with $H\left(\left\{n_{k}\right\}\right)=\lambda \sum_{k} n_{k}+\mu \sum_{k} k n_{k}$, where $\lambda$ and $\mu$ are the Lagrange multipliers that keep the constraints in place. This ensemble is mathematically analogous to a simple Bose gas with energy levels given by $k$. The partition function is given by

$$
Z=\sum_{\left\{n_{k}\right\}} e^{-\lambda \sum_{k} n_{k}-\mu \sum_{k} k n_{k}}=\prod_{k} Z_{k},
$$

with

$$
Z_{k}=\left[1-e^{-\lambda-\mu k}\right]^{-1} .
$$

The average counts are given by $\left\langle n_{k}\right\rangle=-\partial \ln Z_{k} / \partial \lambda=$ $[\exp (\lambda+\mu k)-1]^{-1}$, and the parameters $\lambda$ and $\mu$ are determined via the imposed constraints,

$$
\begin{aligned}
& \sum_{k=0}^{\infty}[\exp (\lambda+\mu k)-1]^{-1}=N, \\
& \sum_{k=0}^{\infty} k[\exp (\lambda+\mu k)-1]^{-1}=E .
\end{aligned}
$$


Further analytical progress can be made by replacing the sums with integrals and using the polylogarithm function and its connection with the Bose-Einstein distribution, $\operatorname{Li}_{s}(z)=\Gamma(s)^{-1} \int_{0}^{\infty}\left(t^{s-1} / e^{t} / z-1\right) \mathrm{d} t$,

$$
\begin{gathered}
\int_{0}^{\infty} d k[\exp (\lambda+\mu k)-1]^{-1}=\frac{\operatorname{Li}_{1}\left(e^{-\lambda}\right)}{\mu}=N, \\
\int_{0}^{\infty} d k k[\exp (\lambda+\mu k)-1]^{-1}=\frac{\operatorname{Li}_{2}\left(e^{-\lambda}\right)}{\mu^{2}}=E .
\end{gathered}
$$

Equation (C5) can be inverted as $e^{-\lambda}=1-\exp (-N / \mu)$, but Eq. (C6) cannot be solved for $\lambda$ in closed form. However, by assuming a sufficiently "high-temperature" regime where $\mu \sim O(1)$, we have that the fugacity simplifies in the thermodynamic limit, $e^{-\lambda} \rightarrow 1$ for $N \gg 1$, and hence we obtain $\mu \simeq \sqrt{\mathrm{Li}_{2}(1) / E}$. Using Eqs. (C5) and (C6), we can write the entropy of the ensemble $\ln \Xi=-\sum_{k}\left[\partial \ln Z_{k} / \partial \lambda+\partial \ln Z_{k} / \partial \mu+\ln Z_{k}\right]$ as

$$
\ln \Xi=\lambda N+2 \mu E,
$$

and for the regime $e^{-\lambda} \rightarrow 1$, we have

$$
\ln \Xi \simeq 2 \sqrt{\zeta(2) E},
$$

where the identity $\mathrm{Li}_{2}(1)=\zeta(2)$ was used, with $\zeta(x)$ being the Riemann zeta function. Although Eq. (C8) becomes asymptotically exact in the thermodynamic limit with $E \sim N$ and $N \gg 1$, the exact solution can also be obtained with arbitrary precision simply by iterating Eqs. (C5) and (C6) as $\hat{\lambda}(t+1)=1-\exp (-N / \mu(t)), \mu(t+1)=$ $\sqrt{E / \operatorname{Li}_{2}(\hat{\lambda}(t))}$, where $\hat{\lambda} \equiv e^{-\lambda}$, with the starting points $\hat{\lambda}(0)=1, \mu(0)=\sqrt{\mathrm{Li}_{2}(1) / E}$, until sufficient convergence is reached, and the results are substituted in Eq. (C7). (We actually use this more precise procedure when computing Eq. (13) in the main text, throughout the analysis.)

[1] M. E. J. Newman, Communities, Modules and Large-Scale Structure in Networks, Nat. Phys. 8, 25 (2011).

[2] S. Fortunato, Community Detection in Graphs, Phys. Rep. 486, 75 (2010).

[3] P. Holme, Core-Periphery Organization of Complex Networks, Phys. Rev. E 72, 046111 (2005).

[4] M. P. Rombach, M. A. Porter, J. H. Fowler, and P. J. Mucha, Core-Periphery Structure in Networks, arXiv:1202.2684.

[5] D. B. Larremore, A. Clauset, and A. Z. Jacobs, Efficiently Inferring Community Structure in Bipartite Networks, Phys. Rev. E 90, 012805 (2014).

[6] A. Clauset, C. Moore, and M. E. J. Newman, Hierarchical Structure and the Prediction of Missing Links in Networks, Nature (London) 453, 98 (2008).
[7] T.P. Peixoto, Hierarchical Block Structures and High-Resolution Model Selection in Large Networks, Phys. Rev. X 4, 011047 (2014).

[8] R. Guimerá and M. Sales-Pardo, Missing and Spurious Interactions and the Reconstruction of Complex Networks, Proc. Natl. Acad. Sci. U.S.A. 106, 22073 (2009).

[9] S. V. Buldyrev, R. Parshani, G. Paul, H. E. Stanley, and S. Havlin, Catastrophic Cascade of Failures in Interdependent Networks, Nature (London) 464, 1025 (2010).

[10] A. Apolloni, C. Poletto, J. J. Ramasco, P. Jensen, and V. Colizza, Metapopulation Epidemic Models with Heterogeneous Mixing and Travel Behaviour, Theor. Biol. Med. Model. 11, 3 (2014).

[11] R. Guimerá and L. A. N. Amaral, Functional Cartography of Complex Metabolic Networks, Nature (London) 433, 895 (2005).

[12] M. E. J. Newman, Modularity and Community Structure in Networks, Proc. Natl. Acad. Sci. U.S.A. 103, 8577 (2006).

[13] M. Girvan and M. E. J. Newman, Community Structure in Social and Biological Networks, Proc. Natl. Acad. Sci. U.S.A. 99, 7821 (2002).

[14] Y.-Y. Ahn, J. P. Bagrow, and S. Lehmann, Link Communities Reveal Multiscale Complexity in Networks, Nature (London) 466, 761 (2010).

[15] G. Palla, I. Derényi, I. Farkas, and T. Vicsek, Uncovering the Overlapping Community Structure of Complex Networks in Nature and Society, Nature (London) 435, 814 (2005).

[16] M. Rosvall and C. T. Bergstrom, Maps of Random Walks on Complex Networks Reveal Community Structure, Proc. Natl. Acad. Sci. U.S.A. 105, 1118 (2008).

[17] P. W. Holland, K. B. Laskey, and S. Leinhardt, Stochastic Blockmodels: First Steps, Soc. Networks 5, 109 (1983).

[18] E. M. Airoldi, D. M. Blei, S. E. Fienberg, and E. P. Xing, Mixed Membership Stochastic Blockmodels, J. Mach. Learn. Res. 9, 1981 (2008).

[19] B. Karrer and M. E. J. Newman, Stochastic Blockmodels and Community Structure in Networks, Phys. Rev. E 83, 016107 (2011).

[20] B. Ball, B. Karrer, and M. E. J. Newman, Efficient and Principled Method for Detecting Communities in Networks, Phys. Rev. E 84, 036103 (2011).

[21] A. Lancichinetti, S. Fortunato, and J. Kertész, Detecting the Overlapping and Hierarchical Community Structure in Complex Networks, New J. Phys. 11, 033015 (2009).

[22] G. Palla, L. Lovász, and T. Vicsek, Multifractal Network Generator, Proc. Natl. Acad. Sci. U.S.A. 107, 7640 (2010).

[23] J. Leskovec, D. Chakrabarti, J. Kleinberg, C. Faloutsos, and Z. Ghahramani, Kronecker Graphs: An Approach to Modeling Networks, J. Mach. Learn. Res. 11, 985 (2010).

[24] M. Mariadassou, S. Robin, and C. Vacher, Uncovering Latent Structure in Valued Graphs: A Variational Approach, Ann. Appl. Stat. 4, 715 (2010).

[25] C. Aicher, A. Z. Jacobs, and A. Clauset, Learning Latent Block Structure in Weighted Networks, J. Complex Networks (2014).

[26] B. Ball and M. E. J. Newman, Friendship Networks and Social Status, Netw. Sci. 1, 16 (2013). 
[27] M. Kivelä, A. Arenas, M. Barthelemy, J. P. Gleeson, Y. Moreno, and M. A. Porter, Multilayer Networks, J. Complex Networks 2, 203 (2014).

[28] W. Fu, L. Song, and E. P. Xing, Dynamic Mixed Membership Blockmodel for Evolving Networks, in Proceedings of the 26th Annual International Conference on Machine Learning (ACM, New York, NY, 2009), pp. 329-336.

[29] X. Yan, C. Shalizi, J. E. Jensen, F. Krzakala, C. Moore, L. Zdeborová, P. Zhang, and Y. Zhu, Model Selection for Degree-Corrected Block Models, J. Stat. Mech. (2014) P05007.

[30] M. E. J. Newman, Community Detection and Graph Partitioning, Europhys. Lett. 103, 28003 (2013).

[31] M. E. J. Newman, Spectral Methods for Community Detection and Graph Partitioning, Phys. Rev. E 88, 042822 (2013).

[32] R. R. Nadakuditi and M. E. J. Newman, Graph Spectra and the Detectability of Community Structure in Networks, Phys. Rev. Lett. 108, 188701 (2012).

[33] F. Krzakala, C. Moore, E. Mossel, J. Neeman, A. Sly, L. Zdeborová, and P. Zhang, Spectral Redemption in Clustering Sparse Networks, Proc. Natl. Acad. Sci. U.S.A. 110, 20935 (2013).

[34] A. Lancichinetti, S. Fortunato, and F. Radicchi, Benchmark Graphs for Testing Community Detection Algorithms, Phys. Rev. E 78, 046110 (2008).

[35] A. Lancichinetti and S. Fortunato, Benchmarks for Testing Community Detection Algorithms on Directed and Weighted Graphs with Overlapping Communities, Phys. Rev. E 80, 016118 (2009).

[36] A. Lancichinetti and S. Fortunato, Community Detection Algorithms: A Comparative Analysis, Phys. Rev. E 80, 056117 (2009).

[37] B. Karrer, E. Levina, and M. E. J Newman, Robustness of Community Structure in Networks, Phys. Rev. E 77, 046119 (2008).

[38] P. D. Grünwald, The Minimum Description Length Principle (MIT Press, Cambridge, 2007).

[39] J. Rissanen, Information and Complexity in Statistical Modeling, 1st ed. (Springer, New York, 2010).

[40] M. Rosvall, A. V. Esquivel, A. Lancichinetti, J. D. West, and R. Lambiotte, Memory in Network Flows and Its Effects on Spreading Dynamics and Community Detection, Nat. Commun. 5, 4630 (2014).

[41] G. Bianconi, Entropy of Network Ensembles, Phys. Rev. E 79, 036114 (2009).

[42] T. P. Peixoto, Entropy of Stochastic Blockmodel Ensembles, Phys. Rev. E 85, 056122 (2012).

[43] M. Rosvall and C. T. Bergstrom, An Information-Theoretic Framework for Resolving Community Structure in Complex Networks, Proc. Natl. Acad. Sci. U.S.A. 104, 7327 (2007).

[44] S. E. Fienberg, M. M. Meyer, and S. S. Wasserman, Statistical Analysis of Multiple Sociometric Relations, J. Am. Stat. Assoc. 80, 51 (1985).

[45] K. Faust and S. Wasserman, Blockmodels: Interpretation and Evaluation, Soc. Networks 14, 5 (1992).

[46] C. J. Anderson, S. Wasserman, and K. Faust, Building Stochastic Blockmodels, Soc. Networks 14, 137 (1992).
[47] P. Latouche, E. Birmelé, and C. Ambroise, Model Selection in Overlapping Stochastic Block Models, Electronic J. Stat. 8, 762 (2014).

[48] T. P. Peixoto, Parsimonious Module Inference in Large Networks, Phys. Rev. Lett. 110, 148701 (2013).

[49] E. T. Jaynes, Probability Theory: The Logic of Science, edited by G. L. Bretthorst (Cambridge University Press, Cambridge, England; New York, NY, 2003).

[50] Sir H. Jeffreys, The Theory of Probability (Oxford University Press, Oxford, 1998).

[51] D. E. Knuth, The Stanford GraphBase: A Platform for Combinatorial Computing, 1st ed. (Addison-Wesley Professional, New York, N.Y.; Reading, MA, 1993).

[52] T.S. Evans, American College Football Network Files, FigShare (2012), 10.6084/m9.figshare.93179.

[53] L. A. Adamic and N. Glance, The Political Blogosphere and the 2004 U.S. Election: Divided They Blog, in Proceedings of the 3rd International Workshop on Link Discovery (ACM, New York, NY, 2005), pp. 36-43.

[54] D. Holten, Hierarchical Edge Bundles: Visualization of Adjacency Relations in Hierarchical Data, IEEE Trans. Visual. Comput. Graph. 12, 741 (2006).

[55] J. Mcauley and J. Leskovec, Discovering Social Circles in Ego Networks, ACM Trans. Knowl. Discov. Data 8, 4:1 (2014).

[56] E. Cho, S. A. Myers, and J. Leskovec, Friendship and Mobility: User Movement in Location-Based Social Networks, in Proceedings of the 17th ACM SIGKDD International Conference on Knowledge Discovery and Data Mining (ACM, New York, NY, 2011), pp. 1082-1090.

[57] D. J. Watts and S.H. Strogatz, Collective Dynamics of "Small-World" Networks, Nature (London) 393, 440 (1998).

[58] J. Leskovec, J. Kleinberg, and C. Faloutsos, Graph Evolution: Densification and Shrinking Diameters, ACM Trans. Knowl. Discov. Data 1, 2 (2007).

[59] M. E. J. Newman, Finding Community Structure in Networks Using the Eigenvectors of Matrices, Phys. Rev. E 74, 036104 (2006).

[60] K. I. Goh, M. E. Cusick, D. Valle, B. Childs, M. Vidal, and A. L. Barabási, The Human Disease Network, Proc. Natl. Acad. Sci. U.S.A. 104, 8685 (2007).

[61] W. W. Zachary, An Information Flow Model for Conflict and Fission in Small Groups, Journal of anthropological research 33, 452 (1977).

[62] J. Leskovec, K. J. Lang, A. Dasgupta, and M. W. Mahoney, Community Structure in Large Networks: Natural Cluster Sizes and the Absence of Large Well-Defined Clusters, arXiv:0810.1355.

[63] B. Klimt and Y. Yang, Introducing the Enron Corpus, in Conference on Email and Anti-Spam, Mountain View, CA, USA (2004).

[64] D. Lusseau, K. Schneider, O. J. Boisseau, P. Haase, E. Slooten, and S.M. Dawson, The Bottlenose Dolphin Community of Doubtful Sound Features a Large Proportion of Long-Lasting Associations, Behav. Ecol. Sociobiol. 54, 396 (2003).

[65] O. Richters and T. P. Peixoto, Trust Transitivity in Social Networks, PLoS One 6, e18384 (2011). 
[66] M. Richardson, R. Agrawal, and P. Domingos, Trust Management for the Semantic Web, in The Semantic Web-ISWC 2003, Lecture Notes in Computer Science No. 2870, edited by D. Fensel, K. Sycara, and J. Mylopoulos (Springer, Berlin-Heidelberg, 2003), pp. 351-368.

[67] H. Yu et al., High-Quality Binary Protein Interaction Map of the Yeast Interactome Network, Science 322, 104 (2008).

[68] T. Reguly, A. Breitkreutz, L. Boucher, B.-J. Breitkreutz, G. C. Hon, C. L. Myers, A. Parsons, H. Friesen, R. Oughtred, A. Tong, C. Stark, Y. Ho, D. Botstein, B. Andrews, C. Boone, O. G. Troyanskya, T. Ideker, K. Dolinski, N. N. Batada, and M. Tyers, Comprehensive Curation and Analysis of Global Interaction Networks in Saccharomyces Cerevisiae, J. Biol. 5, 11 (2006).

[69] S. R. Collins, P. Kemmeren, X.-C. Zhao, J. F. Greenblatt, F. Spencer, F. C. P. Holstege, J. S. Weissman, and N. J. Krogan, Toward a Comprehensive Atlas of the Physical Interactome of Saccharomyces Cerevisiae, Mol. Cell. Proteomics 6, 439 (2007).

[70] H. Salgado et al., RegulonDB v8.0: Omics Data Sets, Evolutionary Conservation, Regulatory Phrases, CrossValidated Gold Standards and More, Nucleic Acids Res. 41, D203 (2013).

[71] J. McAuley and J. Leskovec, Image Labeling on a Network: Using Social-Network Metadata for Image Classification, in Computer Vision-ECCV 2012, Lecture Notes in Computer Science No. 7575, edited by A. Fitzgibbon, S. Lazebnik, P. Perona, Y. Sato, and C. Schmid (Springer, Berlin-Heidelberg, 2012), pp. 828-841.

[72] J. Leskovec, K. J. Lang, A. Dasgupta, and M. W. Mahoney, Community Structure in Large Networks: Natural Cluster Sizes and the Absence of Large Well-Defined Clusters, Internet Math. 6, 29 (2009).

[73] J. Yang and J. Leskovec, Defining and Evaluating Network Communities Based on Ground-Truth, in Proceedings of the ACM SIGKDD Workshop on Mining Data Semantics (ACM, New York, NY, 2012), pp. 3:1-3:8.

[74] R. Albert, H. Jeong, and A.-L. Barabási, Internet: Diameter of the World-Wide Web, Nature (London) 401, 130 (1999).

[75] J. Leskovec, D. Huttenlocher, and J. Kleinberg, Signed Networks in Social Media, in Proceedings of the SIGCHI Conference on Human Factors in Computing Systems (ACM, New York, NY, 2010), pp. 1361-1370.

[76] J. Leskovec, D. Huttenlocher, and J. Kleinberg, Predicting Positive and Negative Links in Online Social Networks, in Proceedings of the 19th International Conference on World Wide Web (ACM, New York, NY, 2010), pp. 641-650.
[77] T.S. Keshava Prasad et al., Human Protein Reference Database-2009 Update, Nucleic Acids Res. 37, D767 (2009).

[78] M. E. J. Newman, Fast Algorithm for Detecting Community Structure in Networks, Phys. Rev. E 69, 066133 (2004).

[79] A. Clauset, M. E. J. Newman, and C. Moore, Finding Community Structure in Very Large Networks, Phys. Rev. E 70, 066111 (2004).

[80] A. Arenas, L. Danon, A. Díaz-Guilera, P. M. Gleiser, and R. Guimerá, Community Analysis in Social Networks, Eur. Phys. J. B 38, 373 (2004).

[81] T. P. Peixoto, Efficient Monte Carlo and Greedy Heuristic for the Inference of Stochastic Block Models, Phys. Rev. E 89, 012804 (2014).

[82] N. Metropolis, A. W. Rosenbluth, M. N. Rosenbluth, A. H. Teller, and E. Teller, Equation of State Calculations by Fast Computing Machines, J. Chem. Phys. 21, 1087 (1953).

[83] W. K. Hastings, Monte Carlo Sampling Methods Using Markov Chains and Their Applications, Biometrika 57, 97 (1970).

[84] A. Decelle, F. Krzakala, C. Moore, and L. Zdeborová, Inference and Phase Transitions in the Detection of Modules in Sparse Networks, Phys. Rev. Lett. 107, 065701 (2011).

[85] A. Decelle, F. Krzakala, C. Moore, and L. Zdeborová, Asymptotic Analysis of the Stochastic Block Model for Modular Networks and Its Algorithmic Applications, Phys. Rev. E 84, 066106 (2011).

[86] P. K. Gopalan and D. M. Blei, Efficient Discovery of Overlapping Communities in Massive Networks, Proc. Natl. Acad. Sci. U.S.A. 110, 14534 (2013).

[87] T. P. Peixoto, The Graph-Tool Python Library, Figshare, doi: 10.6084/m9.figshare.1164194 (2014).

[88] A. V. Esquivel and M. Rosvall, Compression of Flow Can Reveal Overlapping-Module Organization in Networks, Phys. Rev. X 1, 021025 (2011).

[89] P. D. Hoff, A. E. Raftery, and M. S. Handcock, Latent Space Approaches to Social Network Analysis, J. Am. Stat. Assoc. 97, 1090 (2002).

[90] J. Parkkinen, J. Sinkkonen, A. Gyenge, and S. Kaski, A Block Model Suitable for Sparse Graphs, in Proceedings of the 7th International Workshop on Mining and Learning with Graphs (MLG 2009), Leuven (2009).

[91] J. Yang and J. Leskovec, Community-Affiliation Graph Model for Overlapping Network Community Detection, in Proceedings of the 2012 IEEE 12th International Conference on Data Mining (ICDM), (IEEE, Brussels, 2012) pp. 1170-1175. 